\title{
Evolutionary Diversification of Alanine Transaminases in Yeast: Catabolic Specialization and Biosynthetic Redundancy
}

\begin{abstract}
Ximena Escalera-Fanjul, Carlos Campero-Basaldua, Maritrini Colón, James González, Dariel Márquez and Alicia González*
\end{abstract}

Instituto de Fisiología Celular, Departamento de Bioquímica y Biología Estructural, Universidad Nacional Autónoma de México, Mexico City, Mexico

Gene duplication is one of the major evolutionary mechanisms providing raw material for the generation of genes with new or modified functions. The yeast Saccharomyces cerevisiae originated after an allopolyploidization event, which involved mating between two different ancestral yeast species. SCALT1 and SCALT2 codify proteins with 65\% identity, which were proposed to be paralogous alanine transaminases. Further analysis of their physiological role showed that while SCALT1 encodes an alanine transaminase which constitutes the main pathway for alanine biosynthesis and the sole pathway for alanine catabolism, ScAlt2 does not display alanine transaminase activity and is not involved in alanine metabolism. Moreover, phylogenetic studies have suggested that SCALT1 and SCALT2 come from each one of the two parental strains which gave rise to the ancestral hybrid. The present work has been aimed to the understanding of the properties of the ancestral type Lacchancea kluyveri LKALT1 and Kluyveromyces lactis KIALT1, alanine transaminases in order to better understand the SCALT1 and SCALT2 evolutionary history. These ancestral -type species were chosen since they harbor ALT1 genes, which are related to SCALT2. Presented results show that, although $\angle K A L T 1$ and KIALT1 constitute SCALT1 orthologous genes, encoding alanine transaminases, both yeasts display LKAlt1 and KIAlt1 independent alanine transaminase activity and additional unidentified alanine biosynthetic and catabolic pathway(s). Furthermore, phenotypic analysis of null mutants uncovered the fact that KIAlt1 and LKAlt1 have an additional role, not related to alanine metabolism but is necessary to achieve wild type growth rate. Our study shows that the ancestral alanine transaminase function has been retained by the SCALT1 encoded enzyme, which has specialized its catabolic character, while losing the alanine independent role observed in the ancestral type enzymes. The fact that ScAlt2 conserves 64\% identity with LkAlt1 and 66\% with KIAlt1, suggests that ScAlt2 diversified after the ancestral hybrid was formed. SCALT2 functional diversification resulted in loss of both alanine transaminase activity and the additional alanine-independent $L k$ Alt1 function, since SCALT2 did not complement the $L k a l t 1 \Delta$ phenotype. It can be concluded that $L K A L T 1$ and $K I L A L T 1$ functional role as alanine transaminases was delegated to SCALT1, while SCALT2 lost this role during diversification.

Keywords: orthologous genes, alanine metabolism, functional diversification, alanine transaminase, transcriptional regulation 


\section{INTRODUCTION}

Gene duplication plays a central role in evolution, enhancing organism robustness, and providing material for the emergence of new or specialized functions. Different models have been posed to explain which is the possible fate of duplicated genes. The genedosage amplification model considers that gene doubling is not followed by functional diversification and that after duplication both gene copies are retained, augmenting the ancestral function, which could represent a selective advantage (Kondrashov et al., 2002; Kondrashov and Kondrashov, 2006; Tang and Amon, 2013). Conversely, three models including functional divergence have been developed; loss of function model sustains that as a result of the accumulation of loss-of-function mutations one gene copy is lost, becoming a pseudogene (Conrad and Antonarakis, 2007). Alternatively, the neofunctionalization model considers that one of the copies conserves the ancestral function, while the other one acquires a new adaptative function by the accumulation of neutral mutations (Ohno, 1970). And finally, the subfunctionalization model proposes that the accumulation of degenerative mutations split or specialize the ancestral function(s) between the two paralogous, therefore both copies are necessary to perform the function which in the ancestor was carried out by a single gene (Hughes, 1994; Innan and Kondrashov, 2010; Sikosek et al., 2012; Espinosa-Cantú et al., 2015).

For many years, it was accepted that the Saccharomyces cerevisiae (S. cerevisiae) lineage arose from a Whole Genome Duplication (WGD), making this yeast an interesting model to study diversification of paralogous genes (Wolfe and Shields, 1997; Kellis et al., 2004). Recently, a phylogenetic study found compelling evidence indicating that $S$. cerevisiae lineage arose from an interspecies hybridization between one strain related to the Kluyveromyces, Lachancea and Eremothecium (KLE) clade and another one related to Zygosaccharomyces rouxii and Torulaspora delbrueckii $(Z T)$. Although whether the hybrid was the result of the fusion of two diploid cells or two haploid cells that underwent a WGD, is still an open question, both scenarios result in the formation of an allotetraploid with two copies of every gene. After the allotetraploid was formed, intragenic recombinations, full gene conversion, differential gene loss and selection pressures shaped S. cerevisiae genome to the one we observe today, harboring conserved blocks of duplicated genes (Marcet-Houben and Gabaldón, 2015). Thus, the paralogous genes that are observed today can both come from one of the species involved in the formation of the hybrid, or each paralogue could have originated from each one of the parental species which constituted the hybrid. In S. cerevisiae ScALT1 and ScALT2 genes are part of the duplicated blocks coming from the hybridization; however, while ScALT1 comes from the $Z T$ parental strain, ScALT2 parental is closer to the KLE clade, as it can be seen in Figure 1 (Huerta-Cepas et al., 2014).

ScALT1 and ScALT2 are annotated as putative alanine transaminases, however, previous studies of alanine metabolism in S. cerevisiae and ScAlt1 and ScAlt2 physiological roles, showed that when this yeast is grown on glucose and ammonium sulfate as carbon and nitrogen sources, (biosynthetic conditions), alanine is mainly synthesized by the $S c A L T 1$-encoded alanine transaminase, while the ScALT2-encoded protein does not contribute to alanine biosynthesis (Peñalosa-Ruiz et al., 2012). Furthermore, Scalt1 $\Delta$ mutants are not alanine auxotrophs, indicating the existence of a hitherto undescribed alanine biosynthetic pathway, that could be constituted by the previously described glutamine transaminase, which has been proposed to give rise to alanine and $\alpha$-ketoglutaramate, since it uses pyruvate and glutamine as preferred substrates (Soberón and González, 1987), providing an alternative alanine biosynthetic pathway and supporting the observation of a wild type growth phenotype of the Scalt1 $\Delta$ mutant. Worth mentioning is the fact that the glutamine transaminase could not provide an alanine catabolic pathway, since $\alpha$-ketoglutaramate is spontaneously cyclized and used by the $\omega$-amidase to produce $\alpha$-ketoglutarate (Soberón and González, 1987). Accordingly, it was found that the Scalt1 $\Delta$ mutant strain does not grow in the presence of alanine as sole nitrogen source, demonstrating that the ScAlt1 protein constitutes the only pathway for alanine catabolism, and that ScAlt2 does not play a role in either alanine biosynthesis or catabolism. In addition, it was found that ScAlt2 was devoid of alanine transaminase activity, and consequently, ScAlt2 function remains to be discovered (Peñalosa-Ruiz et al., 2012).

The physiological roles and the evolutionary histories of ScALT1 and ScALT2 are appealing, since these genes are inferred to descend from each of the different parental strains involved in the formation of the hybrid that gave rise to the S. cerevisiae lineage, and consequently, it is not possible to tell if at the moment of the hybridization, these two genes, ScALT1 and ScALT2, had the same function or had already functionally diverged. Worth of mention is the fact that, we have previously shown that ScAlt2 does not bear alanine transaminase activity and it might have acquired by either subfunctionalization or neofunctionalization a distinct physiological role. To better understand how SCALT1 and SCALT2 diverged, and the putative ancestral function of $S c A L T 2$ we decided to study the functional role of presumed alanine aminotransferases from pre-WGD yeasts that share a closest ancestor with the parental lineage which carried ScALT2. As such this could be regarded as a proxi to "ancestral type" ScALT2. For this, Lachancea kluyveri (LkALT1) and Kluyveromices lactis (KlALT1), were selected for functional characterization of their encoded enzymes. LkALT1 and KlALT1 were identified by sequence identity, synteny and phylogenetic analysis as the sole orthologous genes of both ScALT1 and ScALT2 present in L. kluyveri and K. lactis, respectively. Both species belong to the so called KLE clade, which is the closest one to ScALT2 parental strain, and share a more distant common ancestor with the ZT clade, from which ScALT1 was originated (Marcet-Houben and Gabaldón, 2015).

Results presented in this paper indicate: (i) LkALT1 and KlALT1 are functionally equivalent to ScALT1 functional orthologous genes, encoding alanine transaminase with a characteristic Ping Pong kinetic mechanism, (ii) while for S. cerevisiae alanine catabolism is strictly ScAlt1-dependent, in L. kluyveri and K. lactis additional unidentified pathways contribute to both alanine biosynthesis and catabolism, (iii) Lkalt1 $\Delta$ and Klalt1 $\Delta$ mutants retain alanine transaminase 


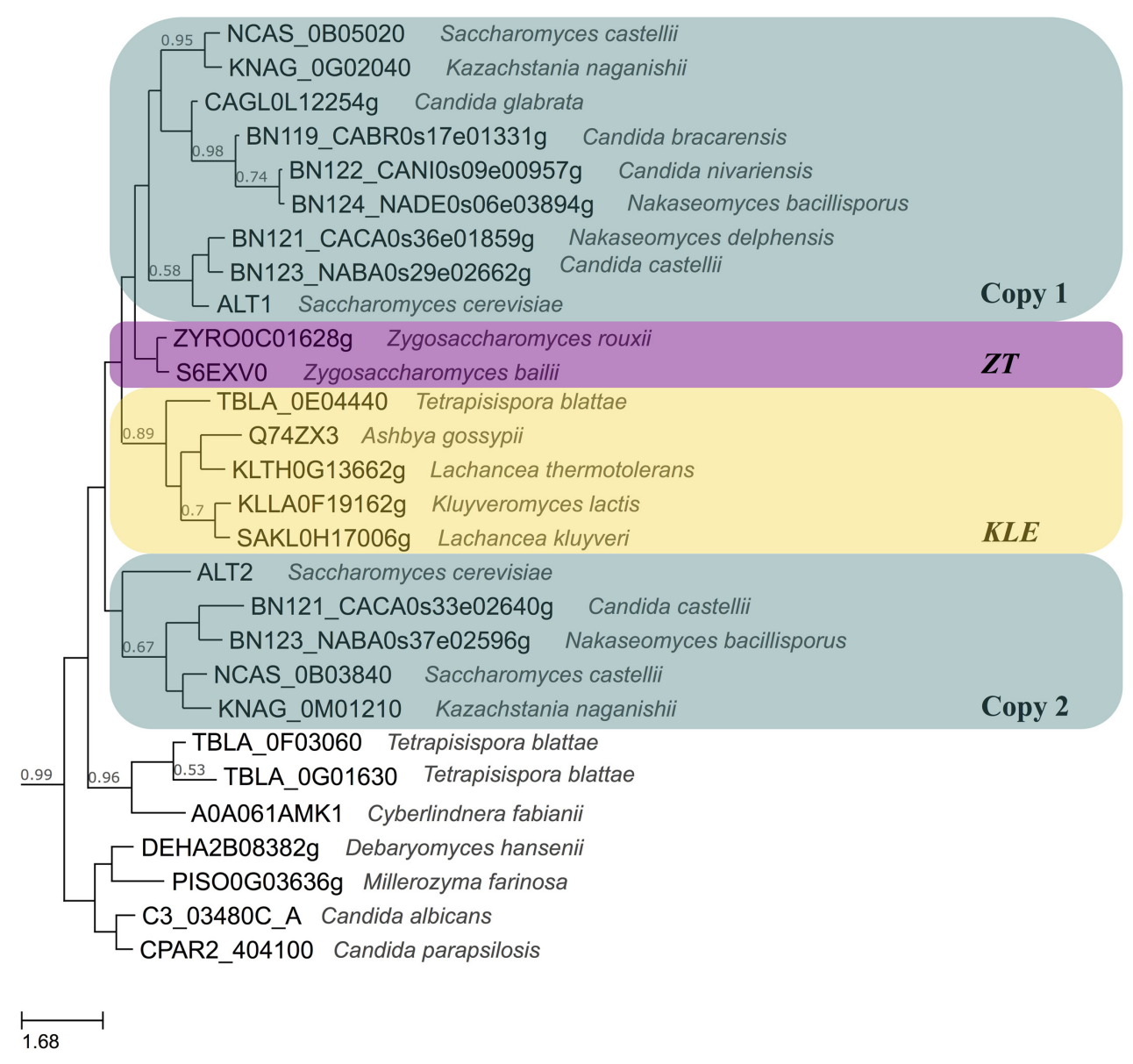

FIGURE 1 | Evolutionary relationships of Alts from yeasts. The phylogeny was modified from http://phylomedb.org alt2 tree in phylome 410. URL: http://phylomedb.org/?q=search_tree\&seqid=alt2

activity indicating the existence of a second alanine transaminase encoding gene in L. kluyveri and K. lactis, (iv) LkAlt1 and KlAlt1 enzymatic activity profile, respectively, follow LkALT1 and KlALT1 expression profile, and v) that as shown by reciprocal complementation tests, LkAlt1 and KlAlt1 have a yet unknown alanine-independent alternative function which neither ScALT1 nor ScALT2 can complement.

\section{MATERIALS AND METHODS}

\section{Strains}

Table 1 describes the characteristics of the strains used in the present work. All strains constructed for this study were derivatives of CLA1-2 (ura3 leu2::LEU2), Lk156-1 (ura3) or Kl155 (ade2 his3 ura3) for S. cerevisiae, L. kluyveri, and K. lactis, respectively. Mutant strains as Scalt1 $\Delta$ (CLA1-2-1), Scalt2 $\Delta$ (CLA1-2-2), Scalt1 $\Delta$ Scalt2 $\Delta$ (CLA1-2-D) (Quezada et al., 2008; García-Campusano, 2009). The L. kluyveri Lkalt1 $\Delta$ (Lk156-2) mutant strain was kindly provided by Dr. Lina Riego, and was obtained by replacing the ORF of LkALT1 with the selectable marker kanMx4. The LkALT1 gene was replaced
TABLE 1 | Saccharomyces cerevisiae, Lacchancea kluyveri, and Kluyveromyces lactis strains used in this work.

\begin{tabular}{|c|c|c|}
\hline Strain & Relevant genotype & Reference \\
\hline CLA1-2 & $\begin{array}{l}\text { MATa ScALT1 ScALT2 ura3 } \\
\text { leu2::LEU2 }\end{array}$ & Quezada et al., 2008 \\
\hline CLA1-2-1 & $\begin{array}{l}\text { MATa Scalt1 } \triangle:: \text { kanMX4 ScALT2 } \\
\text { ura3 leu2::LEU2 }\end{array}$ & García-Campusano, 2009 \\
\hline CLA1-2-2 & $\begin{array}{l}\text { MATa ScALT1 Scalt2 } \triangle:: k a n M X 4 \\
\text { ura3 leu2::LEU2 }\end{array}$ & García-Campusano, 2009 \\
\hline CLA1-2D & $\begin{array}{l}\text { MATa Scalt1 } \triangle:: k a n M X 4 \\
\text { Scalt2 } \Delta:: \text { natMX4 ura3 leu2::LEU2 }\end{array}$ & García-Campusano, 2009 \\
\hline LK 156-1 & Mata $L K A L T 1$ ura3 & $\begin{array}{l}\text { Montalvo-Arredondo et al. } \\
2015\end{array}$ \\
\hline Lk Y156-2 & MATa Lkalt1 $\triangle:: k a n M X 4$ ura3 & This study \\
\hline KI 155 & MAT $\alpha$ ade2 his3 ura3 & Colón et al., 2011 \\
\hline Kl 155-2 & $\begin{array}{l}\text { MATa Klalt1 } \Delta:: \text { kanMX4 ade2 his3 } \\
\text { ura3 }\end{array}$ & This study \\
\hline
\end{tabular}

by homologous recombination using a module containing the kanMX4 cassette (1469 bp) flanked by 1146 bp of $5^{\prime} \mathrm{UTR}$ $(-1181$ to -34$)$ and 1207 bp of $3^{\prime}$ UTR $(+1604$ to +2811$)$ 
sequences of LkALT1. This module (3608 bp) was amplified by overlapped extension PCR with deoxyoligonucleotides X7 and X8 (-1007 to +2061) using a template built up by three independent modules: (i) the LkALT1 5'UTR amplified using the $\mathrm{X} 1$ and $\mathrm{X} 2$ deoxyoligonucleotides and genomic DNA from strain $L k 156-1$ as a template, (ii) the kanMX4 module which was amplified from the pFA6a plasmid using deoxyoligonucleotides $\mathrm{X} 3$ and $\mathrm{X} 4$, and (iii) the LkALT1 3'UTR amplified using deoxyoligonucleotides X5 and X6 and genomic DNA from strain $L k 156-1$ as a template. The PCR product was transformed into the $L k 156-1$ strain. Transformants were selected for G418 resistance $\left(200 \mu \mathrm{g} \mathrm{ml}^{-1}\right)$. Deoxyoligonucleotides $\mathrm{X} 9 / \mathrm{X} 10$ and $\mathrm{X} 11 / \mathrm{X} 12$ were used to verify the construction Lkalt1 $::$ kanMX4, 1547 and 1851 bp (from -1345 of the KlALT1 5'UTR to +216 of kanMX4 and from +874 of kanMX4 to +2879 of $3^{\prime} \mathrm{UTR}$ of KlALT1), respectively. The deoxyoligonucleotides sequences are indicated in Supplementary Table S1.

The K. lactis Klalt1 $\Delta$ (Kl155-2) mutant strain was obtained by replacing the ORF of KlALT1 with the selectable marker kanMX4. The KlALT1 gene was replaced by homologous recombination using a module containing the kanMX4 cassette (1507 bp) flanked by $987 \mathrm{bp}$ of $5^{\prime}$ UTR $(-1169$ to -222$)$ and $1202 \mathrm{bp}$ of $3^{\prime} \mathrm{UTR}(+1587$ to +2771$)$ sequences of KlALT1. This module (2495 bp) was amplified by overlapped extension PCR with deoxyoligonucleotides $(-1169$ to +2771$)$ using a template built up by three independent modules: (i) the KlALT1 5'UTR amplified using the X13 and X14 deoxyoligonucleotides and genomic DNA from strain Kl155 as a template, (ii) the kanMX4 module which was amplified from the pFA6a plasmid using deoxyoligonucleotides X15 and X16, and (iii) the KlALT1 3'UTR amplified using deoxyoligonucleotides $\mathrm{X} 17$ and X18 and genomic DNA from strain Kl155 as a template. The PCR product was transformed into the Kl155 strain. Transformants were selected for G418 resistance (250 $\mu \mathrm{g} \mathrm{ml}^{-1}$ ), the correct module integration was PCR confirmed using the genomic DNA of the G418-resistant transformants as a template and the deoxyoligonucleotides $\mathrm{X} 19$ in combination X20 and X21 in combination X22, these primers generated a module of 1495 and 1978 bp (from 1496 of the KlALT1 $5^{\prime}$ UTR to +216 of kanMX4 and from +1106 of kanMX4 to +3172 of $3^{\prime}$ UTR of KlALT1), respectively. Deoxyoligonucleotides sequences are indicated in Supplementary Table S1.

\section{Growth Conditions}

Strains were routinely grown on minimal medium (MM) containing yeast nitrogen base without amino acids and ammonium sulfate (Difco), glucose ( $2 \% \mathrm{w} / \mathrm{v})$ was used as carbon source, and $40 \mathrm{mM}$ ammonium sulfate or $7 \mathrm{mM}$ alanine were used as the nitrogen source Amino acids needed to satisfy auxotrophic requirements were added at $0.01 \%(w / v)$. Culture growth was monitored by $\mathrm{OD}_{600 \mathrm{~nm}}$ measurements at $30 \mathrm{~m}$ intervals using Bioscreen $\mathrm{C}$ (Oy Growth Curves $\mathrm{Ab}$ Ltd.) set at maximum shaking and $30^{\circ} \mathrm{C}$. Otherwise, cells were incubated at $30^{\circ} \mathrm{C}$ with shaking (250 rpm).

\section{Plasmid Construction}

Cloning into the pRS416 and YEpKD plasmids was performed as follows: to clone the ScALT1 gene into pRS416 plasmid, a 2848 bp region between -931 bp upstream from the start codon and +137 bp downstream from the stop codon was amplified with deoxyoligonucleotides X23 and X24 using genomic DNA from the $S$. cerevisiae (CLA1-2) wild type strain as a template, after PCR amplification, and plasmid digestion with EcoRI and XhoI, cloning was carried out using the NEBuilder HiFi DNA Assembly Master Mix; to clone the ScALT1 gene into YEpKD352 plasmid, a 2619 bp region between -937 bp upstream the start codon and +95 bp downstream the stop codon was amplified with deoxyoligonucleotides X25 and X26, also using genomic DNA from the CLA1-2 wild type strain as template; for the ScALT2 gene, a 2626 bp region between -996 bp upstream from the start codon and +105 bp downstream from the stop codon was amplified with deoxyoligonucleotides X27 and X28 using genomic DNA from the S. cerevisiae (CLA1-2) WT strain as a template; for the LkALT1 gene, a 2611 bp region between -920 bp upstream to the start codon and +281 bp downstream from the stop codon was amplified with deoxyoligonucleotides X29 and X30 using genomic DNA from the L. kluyveri (Lk156-1) wild type strain as a template; and for $K l A L T 1$, a 2619 bp region between -937 bp upstream from the start codon and +95 bp downstream to the stop codon was amplified with deoxyoligonucleotides X31 and X32 using genomic DNA from the K. lactis (Kl155) wild type strain as a template. The PCR products and pRS416 plasmid were digested with restriction enzymes and ligated after gel purification.

\section{Metabolite Extraction and Analysis}

Cell extracts were prepared from exponentially growing cultures. Samples used for intracellular amino acid determination were deproteinized with perchloric acid. Intracellular alanine was determined by HPLC with an Ultrasphere ODS C18 column (Beckman Coulter) with ortho-phthalaldehyde (OPA) derivatization and a mobile phase consisting of a 30 min gradient from 10 to $75 \%$ methanol and $25 \% 0.1 \mathrm{M}$ potassium acetate

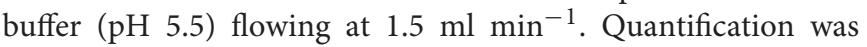
carried out with a fluorescent detector (Quezada et al., 2008).

\section{Cell Extract Preparation and Alanine Transaminase Enzymatic Assay}

Yeast cells were harvested by centrifugation, and washed with cold water. Pellets were suspended in cold extraction buffer (50 mM HEPES, $1 \mathrm{mM}$ PMSF, $1 \mathrm{mM}$ EDTA, $1 \mathrm{mM}$ DTT). For KlAlt1 extraction buffer was supplemented with complete, mini protease inhibitor cocktail, by Roche. Cells were mechanically disrupted with glass beads. The resulting extract was centrifuged to eliminate cellular debris $\left(5,000 \mathrm{rpm}, 4^{\circ} \mathrm{C}, 15 \mathrm{~min}\right)$. The supernatant was recovered, and pyridoxal-5-phosphate was added to a $100 \mu \mathrm{M}$ final concentration. Protein was measured by the method of Lowry (Lowry et al., 1951), using bovine serum albumin as a standard. Enzymatic assay was a modified version from (Graßl and Supp, 1985). The reaction mixture contained reaction buffer (pH7 $100 \mathrm{mM}$ Tris- $\mathrm{HCl}, 400 \mathrm{mM}$ alanine, $24 \mathrm{mM}$ 
$\alpha$-ketoglutarate, $300 \mu \mathrm{M}$ NADH, $40 \mu \mathrm{M}$ pyridoxal-5-phosphate and $5 \mathrm{U} / \mathrm{mL}$ of lactate dehydrogenase). As control, assays were performed without alanine. To determine specific activity the slope obtained from this negative control was subtracted to obtained with the complete assay. All assays were performed at $340 \mathrm{~nm}, 25^{\circ} \mathrm{C}$ in a Varian Cary 50 spectrophotometer.

\section{Cloning and Expression}

The LkALT1 and KlALT1 genes were PCR amplified using the deoxyoligonucleotides pairs X33/X34 and X35/X36, respectively. To amplify LkALT1 genomic DNA of the $L k 156-1$ WT strain was used as template. Alternatively, to amplify KlALT1 genomic DNA of the Kl155 wild type strain genomic DNA was used as template. Obtained PCR product and the pET-28a $(+)$ plasmid were NheI/XhoI digested for LkALT1 cloning and NdeI/XhoI digested for KlALT1 cloning. After gel purification plasmids and inserts were ligated. Ligations were transformed into the $\mathrm{DH} 5 \alpha$ E. coli strain. After plasmid purification, correct cloning was verified by sequencing. For heterologous expression, the Rosetta2 (DE3) E. coli strain was transformed. Selected clones were grown in LB medium supplemented with $50 \mu \mathrm{g} \mathrm{ml} \mathrm{m}^{-1}$ of kanamycin and $70 \mu \mathrm{g} \mathrm{ml}^{-1} 30$ of chloramphenicol, cells were incubated at $37^{\circ} \mathrm{C}$ with shaking $(250 \mathrm{rpm})$. When the cultures reached an $\mathrm{OD}_{600 \mathrm{rmnm}} 0.75$ the expression of the proteins was induced with $100 \mu \mathrm{mol} / \mathrm{L}$ of IPTG (Iso-Propil-Tio-Galactoside), incubated over night at $30^{\circ} \mathrm{C}$ with shaking $(250 \mathrm{rpm})$. IPTG induced cells were harvested by centrifugation at $3750 \mathrm{rpm}$ for $30 \mathrm{~min}$.

\section{Rosetta 2(DE3) Whole Cell Soluble Protein Extract}

Cells were thawed and resuspended in $4 \mathrm{ml}$ of lysis buffer per $100 \mathrm{ml}$ of culture $(10 \mathrm{mmol} / \mathrm{L}$ imidazol, $50 \mathrm{mmol} / \mathrm{L} \mathrm{NaCl}$, and $10 \mathrm{mmo} / \mathrm{L}$ Tris-HCl pH8.0). Protein extracts were obtained by sonication (Ultrasonic Processor Model: VCX 130) with a tip sonicator keeping the tubes on ice; five cycles (60\% amplitude, one second on and one second off for $1 \mathrm{~min}$ ) with $1 \mathrm{~min}$ of incubation on ice between each cycle. After centrifugation at $17000 \mathrm{rpm}$ for $10 \mathrm{~min}$ at $4^{\circ} \mathrm{C}$, the supernatant was recovered, and pyridoxal-5-phosphate was added to a $100 \mu \mathrm{M}$ final concentration.

\section{Affinity Chromatography}

To purify alanine transaminase isozymes, supernatant obtained as stated above, was loaded on an equilibrated nickel column (Ni-NTA Agarose 100, Thermo Fisher Scientific), which was then washed 30 times with $30 \mathrm{mmol} / \mathrm{L}$ imidazol. The protein was eluted with $200 \mathrm{mmol} / \mathrm{L}$ imidazol and $100 \mu \mathrm{M}$ final concentration of pyridoxal-5-phosphate was added. Protein homogeneity was verified by polyacrylamide gel electrophoresis 10\% (SDS-PAGE) stained with Coomassie Blue (Supplementary Figure S1).

\section{Enzyme Kinetics and Analysis of Kinetic Data}

Initial velocity measurements were performed by varying both substrates simultaneously (for LkALT1 alanine from 1.00 to $20.00 \mathrm{mM}$ and $\alpha$-ketoglutarate from 0.05 to $1.50 \mathrm{mM}$; for KlALT1 alanine from 4.00 to $80.00 \mathrm{mM}$ and $\alpha$-ketoglutarate from 0.25 to $5.00 \mathrm{mM}$ ), and the results where globally fitted to the equation for an enzyme with a ping-pong mechanism (Segel, 1975) with the Prism software GraphPad Prism 6.00 (Software Inc.).

\section{Northern Blot Analysis}

Northern blot analysis was carried out as previously described by Struhl and Davis (1981). Total yeast RNA was prepared from $100 \mathrm{ml}$ cultures grown to indicated $\mathrm{OD}_{600 \mathrm{~nm}}$ in $\mathrm{MM}$ with $2 \%$ glucose, ammonium sulfate, or alanine as a nitrogen source. PCR products were used as probes. For $L k A L T 1$, a 400 bp product was amplified with deoxyoligonucleotides X37 and X38; for KlALT1, a 897 bp PCR product was amplified with deoxyoligonucleotides X39 and X40; Lk18s and Kl18s were used as internal loading standards. Both $L k 18 s$ and Kl18s were amplified with deoxyoligonucleotides X41 and X42 a product of 476 bp and 477 bp was amplified for LkALT1 and KlALT1, respectively.

\section{Nucleosome Scanning Assay (NuSA)}

The nucleosome scanning assay was made to see the chromatin organization $L k A L T 1$ and KlALT1 promoter, and the procedure to the study of the positioning of nucleosomes on promoters was made as described by Infante et al. (2011). When the cultures reached an $\mathrm{OD}_{600} \mathrm{~nm}$ of 0.6 at, genetic DNA was obtained of Kl155, and Lk156-1 strains grown on MM with $40 \mathrm{mM}$ ammonium sulfate or $7 \mathrm{mM}$ alanine as nitrogen source and $2 \%$ glucose. Cells were treated with formaldehyde (1\% final concentration) for $20 \mathrm{~min}$ at $37^{\circ} \mathrm{C}$ and then glycine $(125 \mathrm{mmol} / \mathrm{L}$ final concentration) for $5 \mathrm{~min}$ at $37^{\circ} \mathrm{C}$. Formaldehyde-treated cells were harvested by centrifugation, washed with Tris-buffered saline, and then incubated in Buffer Z2 ( $1 \mathrm{~mol} / \mathrm{L}$ Sorbitol, $50 \mathrm{mmol} / \mathrm{L}$ Tris-Cl at $\mathrm{pH} 7.4,10 \mathrm{mmol} / \mathrm{L} \beta$-mercaptoethanol) containing $2.5 \mathrm{mg}$ of zymolase $20 \mathrm{~T}$ for $20 \mathrm{~min}$ at $30^{\circ} \mathrm{C}$ on rocker platform. Spheroplast were pelleted by centrifugation at $3000 \mathrm{~g}$, and resuspended in $1.5 \mathrm{ml}$ of NPS buffer $(0.5 \mathrm{mmol} / \mathrm{L}$ Spermidine, $0.075 \%$ NP-40, $50 \mathrm{mmol} / \mathrm{L} \mathrm{NaCl}, 10 \mathrm{mmol} / \mathrm{L}$ Tris $\mathrm{pH} 7.4,5 \mathrm{mmol} / \mathrm{L} \mathrm{MgCl}_{2}, 1 \mathrm{mmol} / \mathrm{L} \mathrm{CaCl}_{2}, 1 \mathrm{mmol} / \mathrm{L}$ $\beta$-mercaptoethanol). Samples were divided into three $500 \mu \mathrm{l}$ aliquots that were then digested with $22.5 \mathrm{U}$ of MNase (Nuclease S7 from Roche) at $50 \mathrm{~min}$ at $37^{\circ} \mathrm{C}$. Digestions were stopped with $12 \mu \mathrm{l}$ of Stop buffer (50 mmol/L EDTA and 1\% SDS) and were treated with $100 \mu \mathrm{g}$ of proteinase $\mathrm{K}$ at $65^{\circ} \mathrm{C}$ over night. DNA was extracted twice by phenol/chloroform and precipitated with $20 \mu \mathrm{l}$ of $5 \mathrm{~mol} / \mathrm{L} \mathrm{NaCl}$ and equal volume of isopropanol for $30 \mathrm{~min}$ at $-20^{\circ} \mathrm{C}$. Precipitates were resuspended in $40 \mu \mathrm{l}$ of TE and incubated with $20 \mu \mathrm{g}$ RNase $\mathrm{A}$ for $1 \mathrm{~h}$ at $37^{\circ} \mathrm{C}$. DNA digestions were separated by gel electrophoresis from a $1.5 \%$ agarose gel. Monosomal bands (150 bp) were cut and purified by Wizard SV Gel Clean-Up System Kit (Promega, REF A9282). DNA samples were diluted 1:30 and used in quantitative polymerase chain reactions (qPCR) to quantify the relative MNase protection of each $L k A L T 1$ and KlALT1 template. qPCR analysis was performed using a Corbett Life Science Rotor Gene 6000 machine. The detection dye used was SYBR Green $(2 \times$ KAPA SYBR FAST qBioline and Platinum SYBR Green from Invitrogen). Real-time PCR was carried out as follows: $94^{\circ}$ for 
5 min ( 1 cycle), $94^{\circ}$ for $15 \mathrm{~s}, 58^{\circ}$ for $20 \mathrm{~s}$, and $72^{\circ}$ for $20 \mathrm{~s}(35$ cycles). Relative protection was calculated as a ratio to the control $L k V C X 1$, and KlVCX1 template found within a well-positioned nucleosome in +250 bp of the ORFs. The PCR primers amplify from around -950 to $+250 \mathrm{bp}$ (Supplementary Tables S2, S3) of LkALT1 and KlALT1 locus whose coordinates are given relative to the ATG $(+1)$.

\section{Statistical Analysis}

All the experiments were repeated two or three times. Data were analyzed statistically using unpaired $t$-test with Welch's correction using Prism version 7.0c computer software. $P$ values of 0.1 or less were considered statistically significant.

\section{RESULTS}

\section{L. kluyveri LkALT1 and K. lactis KIALT1 Encoded Enzymes Contribute to Alanine Metabolism}

To address whether L. kluyveri LkALT1 and K. lactis KlALT1 preformed similar physiological roles to that of ScAlt1, an analysis of its contribution to alanine metabolism was assessed by phenotypic analysis, through the obtention and phenotypic analysis of pertinent null mutants (Figure 2A). In a biosynthetic medium, with ammonium as nitrogen source, Lkalt1 $\Delta$ displayed a $20 \%$ lower growth rate than the one observed for the wild type strain, surprisingly, alanine addition to the medium did not restore wild type growth rate. In a catabolic condition, when alanine was provided as sole nitrogen source, Lkalt1 $\Delta$ growth rate was near half to that observed for the wild type strain; such growth reduction was not alleviated when ammonium sulfate was added to the growth medium, indicating that the role played by LkAlt1 is not fully restored neither with alanine nor with ammonium sulfate, this observation will be discussed further. Conversely, in a biosynthetic medium $K$. lactis Klalt1 $\Delta$ null mutant displayed wild type phenotype, and the braditrophic phenotype observed on alanine as sole nitrogen source was fully restored in the presence of both ammonium sulfate and alanine. These results indicate that, as well as for ScALT1, the orthologous genes LkALT1 and KlALT1 are involved in alanine catabolism. Nevertheless, unlike in $S$. cerevisiae, in L. kluyveri and $K$. lactis there is an alternative pathway to catabolize alanine. The growth rate displayed by Lkalt1 $\Delta$ and Klalt $1 \Delta$ in a biosynthetic medium suggests that as well as for $S$. cerevisiae, there is an independent pathway(s) for alanine biosynthesis, hampering the possibility to observe LkALT1 and KlALT1 contribution to alanine biosynthesis by growth rate analysis.

To further analyze the contribution of LkALT1 and KlALT1 to alanine biosynthesis, intracellular concentrations of this amino acid were determined in wild type and single mutants Scalt $1 \Delta$, Lkalt1 $\Delta$ and Klalt1 $\Delta$ during early exponential growth phase $\left(\mathrm{OD}_{600 \mathrm{~nm}}\right.$ 0.3-0.6) on biosynthetic medium. To estimate alanine intracellular concentrations, a cell volume of $42 \mathrm{fl}$ was considered for the three strains under study (Jorgensen et al., 2002;

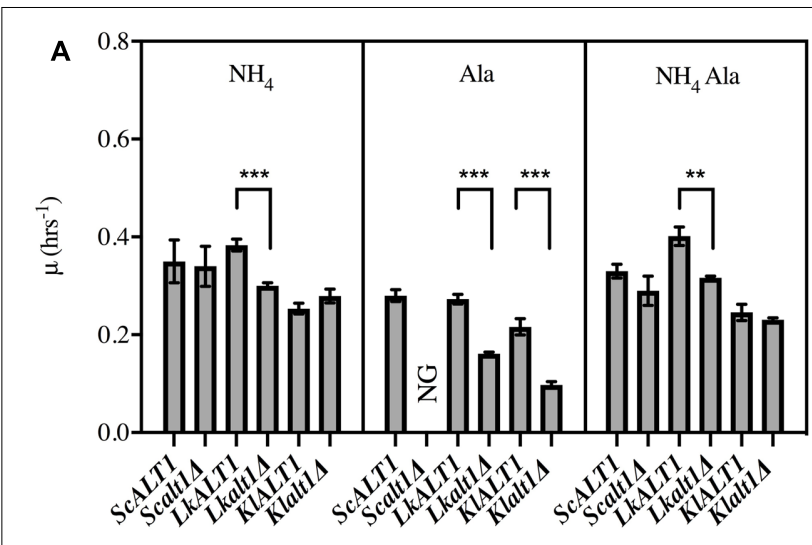

B

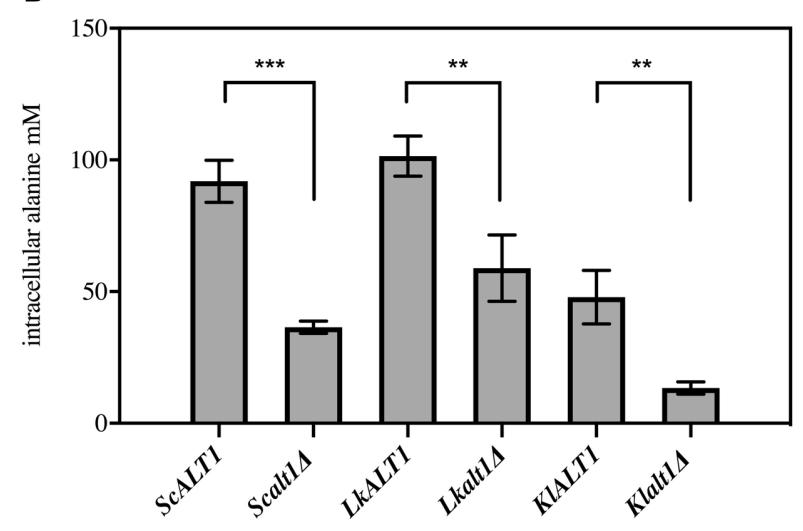

FIGURE 2 | Saccharomyces cerevisiae SCALT1, Lacchancea kluyveri LKALT1, and Kluyveromyces lactis KIALT1 are involved in alanine metabolism. (A) growth rate analysis of S. cerevisiae, L. kluyveri, and K. lactis wild type strain and single mutants was performed using glucose as carbon source and as sole nitrogen source ammonium sulfate, alanine, or ammonium sulfate plus alanine. Specific growth rate was determined during exponential phase. Values are presented as means \pm SD from three independent experiments. $N G$, no growth. The asterisks indicates significantly different $\left(^{*} p<0.1\right.$; ${ }^{* *} p<0.05$; $* p<0.01$ ) compared with the wt control. (B) Intracellular concentration of alanine in extracts obtained from glucose ammonium grown cells. After cells were grown and harvested during exponential phase, cell free extracts were prepared and alanine pools were determined, values are presented as means \pm SD from two or three independent experiments. NG, no growth. The asterisks indicate significantly different $\left({ }^{*} p<0.1 ;{ }^{* *} p<0.05\right.$; $\left.{ }^{*} p<0.01\right)$ compared with the wt control.

Klis et al., 2014). As reported earlier, ScAlt1 contributed with $75-60 \%$ of the alanine pool, whereas the ScAlt1-independent pathway only afforded 25-40\% (Peñalosa-Ruiz et al., 2012). In Lkalt $1 \Delta$ and Klalt $1 \Delta$ strains a decrease of 42 and $70 \%$ in the intracellular alanine concentration, as compared to that found in the pertinent wild type strains, was observed (Figure 2B). In summary, presented results established that LkALT1 and KlALT1 are involved in alanine biosynthesis and catabolism, and that as well as for $S$. cerevisiae, there are alternative pathway(s) involved in alanine biosynthesis, which respectively provide 58 and 30\% of the alanine pool in L. kluyveri and K. lactis, respectively. In regard to alanine catabolism, Lkalt $1 \Delta$ and Klalt $1 \Delta$ achieve $50 \%$ 
growth rate as compared with the one achieved in the wild type strain, indicating that as opposed to what has been observed in S. cerevisiae, in L. kluyveri and K. lactis, there are additional alanine catabolic pathways.

\section{LKALT1 and KIAL1 Are the Functional Orthologs of ScALT1}

Above presented results indicate that ScAlt1, LkAlt1, and KlAlt1 have close physiological roles. To analyze whether the three enzymes have an orthologous function, growth rate analysis of clones obtained from reciprocal complementations was performed (Figure 3). In a catabolic condition, where alanine was present as sole nitrogen source, transformation of an Scalt1 $\triangle$ mutant with plasmids carrying either ScALT1, LkALT1, or KlALT1 restored wild type growth (Figure 3A). Under the same culture conditions, Lkalt1 $\Delta$ mutant strain was only partially complemented with plasmids carrying either ScALT1 or KlALT1, while it was fully complemented when transformed with LkALT1 (Figure 3B). When a Klalt1A mutant strain was transformed with plasmids harboring either ScALT1, LkALT1, or KlALT1 wild type growth was attained (Figure 3C). As expected, ScALT2 was unable to complement neither one of the three mutants tested (Figures 3A-C). This result indicates that LkAlt1 and KlAlt1 catabolic character has been maintained by ScALT1, conversely ScALT2 has completely lost the capacity to fulfill ScALT1, LkALT1, or KlALT1catabolic role.

As shown in Figure 2 the only mutant with a decreased growth rate in a biosynthetic condition was Lkalt1 $\Delta$. When it was transformed with plasmids harboring either LkALT1 or KlALT1 wild type growth rate was attained, but neither ScALT1 nor SCALT2 were able to restore the growth rate observed in the wild type transformed with an empty plasmid (Figure 3B). Scalt $1 \Delta$ and Klalt1 $\Delta$ mutant strains, did not show a growth defect in the presence of ammonium sulfate as sole nitrogen source. In order to analyze their biosynthetic role, alanine pools were determined in extracts prepared from the mutant strains transformed with plasmids bearing the pertinent genes, as controls wild type and mutants transformed with empty plasmids were used (Figure 4), all extracts were prepared from exponential growth phase $\left(\mathrm{OD}_{600} \mathrm{~nm}\right.$ 0.3-0.6). Both ScAlt1 and LkAlt1 were able to restore alanine intracellular wild type concentrations in Scalt1 $\Delta$, Lkalt1 $\Delta$ and Klalt1 $\Delta$ mutant strains (Figures 4A-C). Lkalt $1 \Delta$ and Klalt1 $\Delta$ transformed with KlALT1 displayed a wild type alanine intracellular concentration (Figures 4B,C). Nevertheless, KlAlt1 failed to reestablish the wild type alanine concentration of the Scalt1 $\Delta$ mutant (Figure 4A). The inability of KlAlt1 to restore Scalt1 $\Delta$ alanine pool when grown under biosynthetic conditions could be due to peculiar kinetic properties of KlALT1-encoded protein and/or to a low transcriptional rate of KlALT1. As expected, all mutant strains transformed with plasmids harboring ScALT2 presented an equivalent alanine intracellular concentration to that found in the negative controls.

These results, show that $S c$ Alt1, LkAlt1, and KlAlt1 can fully or partially substitute each other when alanine or ammonium sulfate is present as sole nitrogen source, confirming that ScAlt1 has retained enough ancestral characteristics to conclude that LkAlt1 and KlAlt1 are the functional orthologs of ScAlt1 with respect to its performance in both alanine biosynthesis or catabolism, conversely, ScAlt2 has lost its role in alanine metabolism.

It is important to highlight the observation that in a medium provided with ammonium sulfate as sole nitrogen source Lkalt1 $\Delta$ with or without an empty plasmid displayed a growth defect (Figures 2, 3B) and wild type growth phenotype was not attained when neither alanine was added to the medium (Figure 2) nor when Lkalt1 $\Delta$ was transformed with a plasmid harboring ScALT1 (Figure 3B). Although, Lkalt1 $\Delta$ transformed with ScALT1, displayed wild type alanine intracellular concentration (Figure 4B), indicating that Lkalt1 $\Delta$ growth defect in a biosynthetic condition was not due to a lower alanine intracellular concentration. Taken together these observations provide strong experimental evidence to sustain that LKALT1 has a yet unidentified alternative function that is also present in KlALT1, since plasmids carrying KlALT1 not only restored Lkalt1 $\Delta$ alanine pool (Figure 4B), but also complemented the growth phenotype under the biosynthetic condition tested (Figure 3B). Worth of mention is the fact that in K. lactis, KlALT1 alternative function does not result in any apparent phenotype when Klalt1 $\Delta$ mutant is grown on either ammonium sulfate or alanine as nitrogen sources. The observation that KlALT1 complements Lkalt1 $\Delta$ decreased growth on ammonium sulfate and alanine uncovers the fact that KlAlt1 harbors an equivalent function to that observed in LkAlt1. Furthermore, both ScALT1 and ScALT2 were unable to reestablish wild type growth rate of Lkalt1 $\Delta$ strain in a medium with ammonium sulfate and alanine as nitrogen sources (Figure 3B), indicating that neither ScALT1 nor ScALT2 can substitute the supplementary function present in LkALT1 (Figures 2A, 3B).

\section{ScAlt1, LkAlt1, and KIAlt1 Exhibit Alanine Transaminase Activities}

To compare the ScAlt1, LkAlt1, and KlAlt1 transaminase activities displayed during biosynthetic and catabolic conditions, extracts obtained during exponential phase $\left(\mathrm{OD}_{600} \mathrm{~nm}\right.$ 0.4-0.6) from S. cerevisiae, L. kluyveri, and K. lactis wild type strains, grown in glucose-ammonium sulfate and glucose-alanine, were tested for alanine transaminase activity. Extracts obtained from Scalt $1 \Delta$, Lkalt1 $\Delta$, and Klalt1 $\Delta$ mutant strains were used as negative controls (Table 2). In agreement with a previous report from our group (Peñalosa-Ruiz et al., 2012), ScAlt1 and LkAlt1 alanine transaminase specific activity increased approximately 10 -fold in cultures provided with alanine as sole nitrogen source as compared to those grown on ammonium sulfate, suggesting alanine could play and activator role. In K. lactis alanine transaminase activity was equivalent in samples prepared from cultures grown on either alanine or ammonium sulfate as nitrogen sources. Unexpectedly, alanine transaminase activity was detected in extracts of the mutant strains Lkalt1 $\Delta$ and Klalt $1 \Delta$ grown in either ammonium or alanine as sole nitrogen 


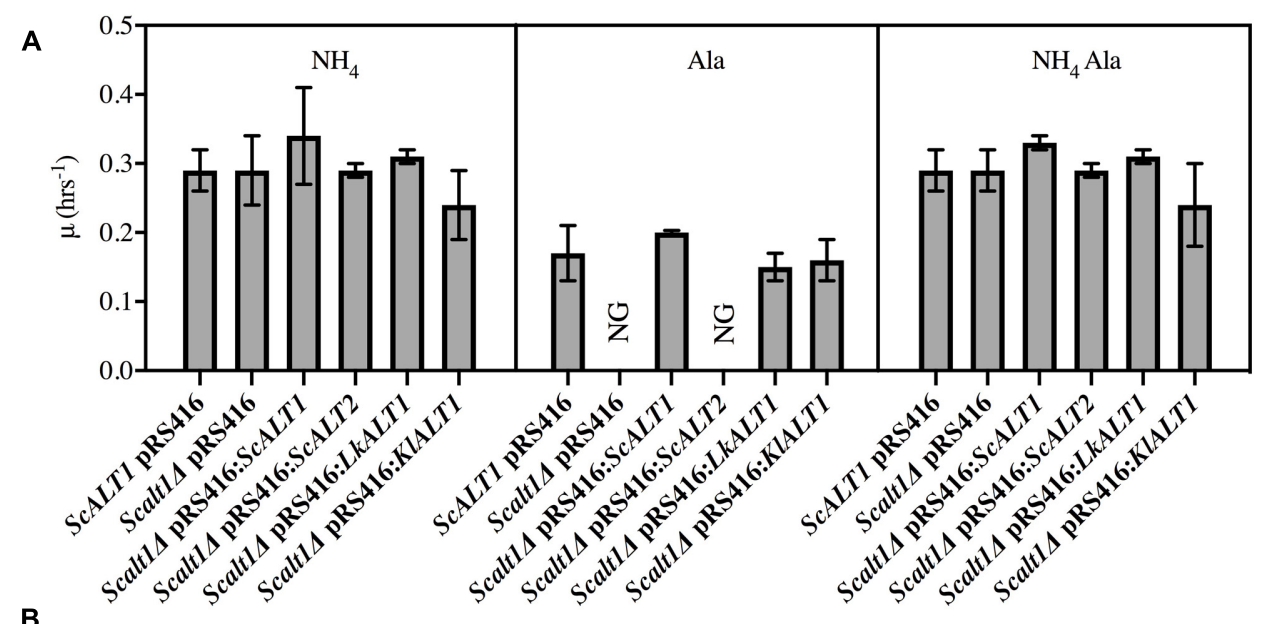

B
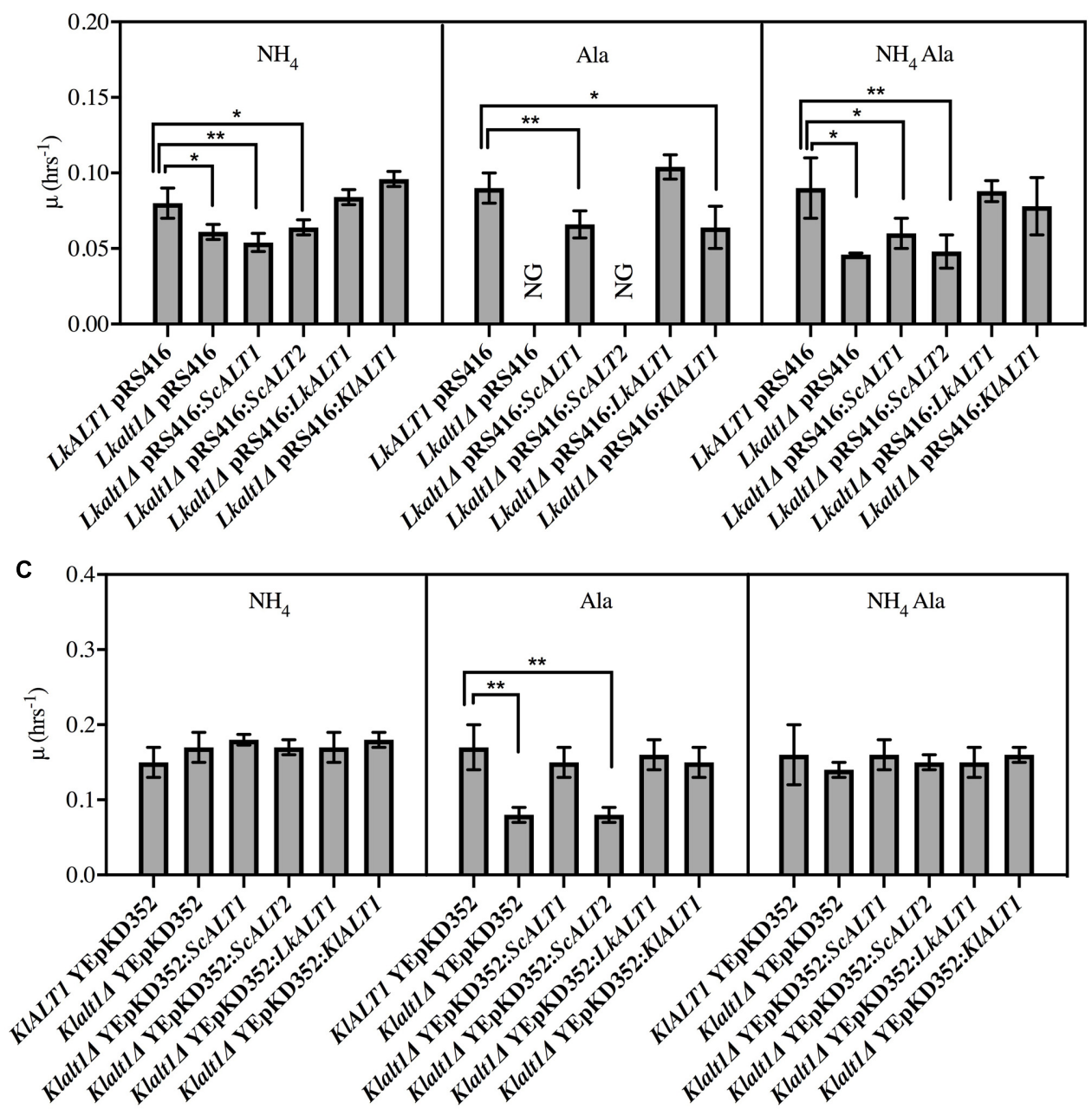

FIGURE 3 | SCALT1, LKALT1, and KIALT1 can substitute each other under catabolic conditions. Reciprocal complementation test was performed using glucose as carbon source and as sole nitrogen source ammonium sulfate, alanine or ammonium sulfate plus alanine. Specific growth rate was determined during exponential phase for (A) S. cerevisiae, (B) L. kluyveri, and (C) K. lactis pertinent transformed cells. For ectopic expression, the plasmid pRS416 was used for S. cerevisiae and L. Kluyveri and the YEpKD352 for $K$. lactis. Values are presented as means \pm SD from three independent experiments. NG, no growth. The asterisks indicate significantly different $\left({ }^{*} p<0.1 ;{ }^{* *} p<0.05 ;{ }^{*} p<0.01\right)$ compared with the wt control. NG, no growth. 

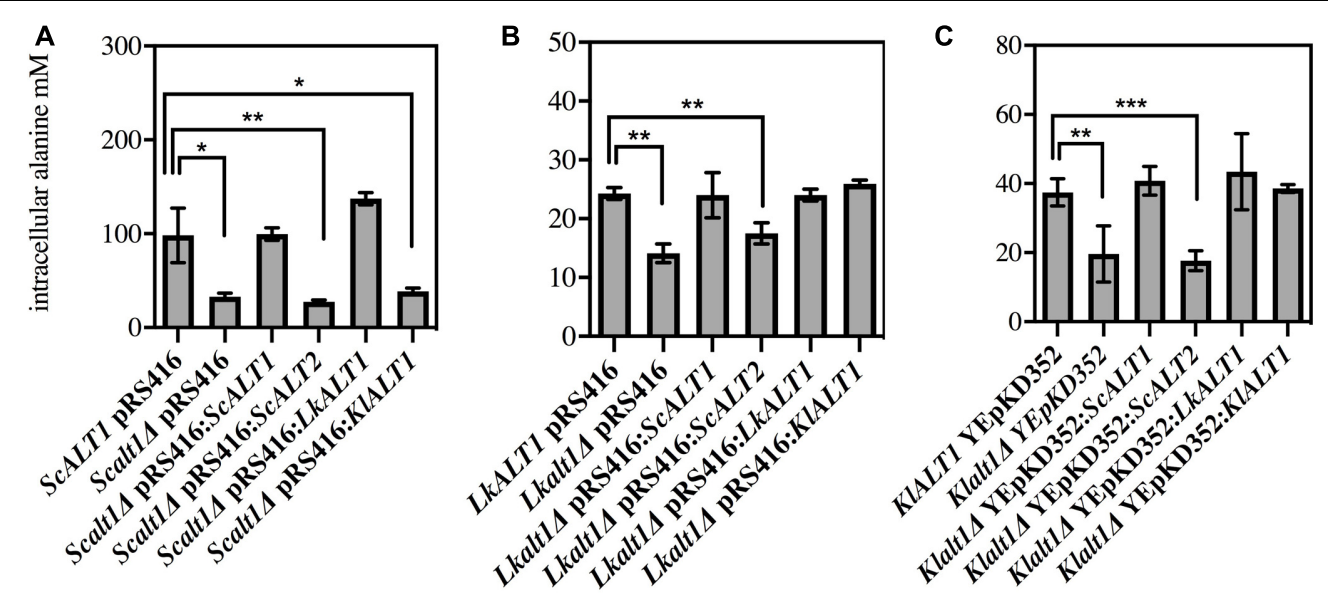

FIGURE 4 | Reciprocal complementation test under biosinthetic conditions. Intracellular concentration of alanine from (A) S. cerevisiae, (B) L. kluyveri and (C) K. lactis transformed strains was obtained from glucose ammonium grown cell extracts. After cells were grown and harvested during exponential phase, cell free extracts were prepared and alanine pools were determined. Values are presented as means \pm SD from two-three independent experiments. The asterisks indicates significantly different $(* p<0.1 ; * p<0.05 ; * p<0.01)$ compared with the wt control.

sources (Table 2). When the extracts came from ammonium sulfate the activity displayed by the mutant Lkalt1 $\Delta$ was similar to that observed in the L. kluyveri wild type strain. However, in extracts prepared from cells grown on alanine as sole nitrogen source, mutant strain Lkalt1 $\Delta$ activity was 20 times lower than that observed in the wild type strain (0.12 vs. $2.77 \mu$ moles $\min ^{-1} \mathrm{mg}$ protein ${ }^{-1}$ ), although it was only 2.5-fold lower to that one observed in extracts from ammonium sulfate ( 0.12 vs. $0.28 \mu$ moles $\min ^{-1} \mathrm{mg}_{\text {protein }}{ }^{-1}$ ), suggesting the existence of an additional alanine transaminase. Similarly, the specific activity detected in extracts from the mutant Klalt $1 \Delta$ coming from ammonium or alanine was smaller than the observed in the wild type $(0.024$ vs. $0.036 \mu$ moles $\min ^{-1} \mathrm{mg}$ protein ${ }^{-1}$ ), again suggesting the existence of a second alanine transaminase in L. kluyveri and $K$. lactis. It can be thus concluded that ScALT1, LkALT1, and KIALT1 encoded proteins display alanine transaminase activity in the three yeasts under study and that L. kluyveri and K. lactis alanine transaminase activity is not exclusively encoded by $L k A L T 1$ and KlALT1.

TABLE 2 | Alanine transaminase specific activity in extracts prepared from glucose-ammonium sulfate or glucose-alanine grown cultures.

\begin{tabular}{lcc}
\hline & \multicolumn{2}{c}{ Glucose } \\
\cline { 2 - 3 } & \multicolumn{1}{c}{$\mathbf{N H}_{\mathbf{4}}$} & L-alanine \\
\hline ScALT1 & $0.093 \pm 0.001$ & $0.821 \pm 0.093$ \\
Scalt1 $\Delta$ & $0.018 \pm 0.001$ & $\mathrm{NG}$ \\
LKALT1 & $0.259 \pm 0.014$ & $2.771 \pm 0.399$ \\
Lkalt1 & $0.289 \pm 0.142$ & $0.120 \pm 0.031$ \\
KIALT1 & $0.036 \pm 0.003$ & $0.036 \pm 003$ \\
Klalt1 & $0.024 \pm 0.003$ & $0.024 \pm 0.002$
\end{tabular}

Specific activity is expressed as $\mu \mathrm{mol} \mathrm{min}^{-1} \mathrm{mg}$ of protein ${ }^{-1}$. No growth (NG). Values are presented as mean from three mesurments $\pm S D$. NG, no growth.

\section{Determination of Alanine Transaminase Activity in Complemented Strains}

To confirm the orthologous function of the three studied enzymes, alanine transaminase activity was also tested in Scalt1 $\Delta$, Lkalt1 $\Delta$ and Klalt1 $\Delta$ mutants transformed with plasmids carrying either ScALT1,ScALT2, LkALT1, or KlALT1 in either glucose ammonium sulfate or glucose alanine, as controls wild type and mutants transformed with empty plasmids were used (Figure 5). In many cases, it was observed that extracts from the mutant strains transformed with plasmids harboring either ScALT1, LkALT1 or KlALT1 exhibited higher specific activity, than the one observed in the wild type strains in each one of the conditions tested (Figures 5A,C,D,F). These results could be attributed to both a difference in the transcriptional regulation of the heterologous enzymes or to the plasmid copy number, since YEpKD352 is a high copy number plasmid and pRS416 is a centromeric (CEN) plasmid, of which one or two copies are maintained per cell (Sikorski and Hieter, 1989), thus, increased enzymatic activity can be expected. As expected the Scalt1 $\Delta$ mutants transformed with plasmids carrying either ScALT1 or LkALT1 displayed higher specific activity than the wild type strain in extracts from either glucose-ammonium or glucose-alanine (Figures $\mathbf{5 A , D}$ ). As in the wild type strain, the specific activity of these transformed strains was higher in extracts coming from alanine supplemented medium than from ammonium sulfate. The specific activity of extracts from Scalt1 $\Delta$ transformed with KlALT1 were not detectable in neither one of the conditions tested (Figures 5A,D). However, as described earlier, plasmids harboring KlALT1 were able to functionally substitute ScALT1 in medium with alanine as sole nitrogen source (Figure 3A), suggesting that lack of alanine transaminase activity in the extracts prepared from this strain grown on either glucose-ammonium sulfate or glucose-alanine was due to a an in vitro artifact which prevents detection of activity in these extracts. When grown in a medium with ammonium sulfate as 

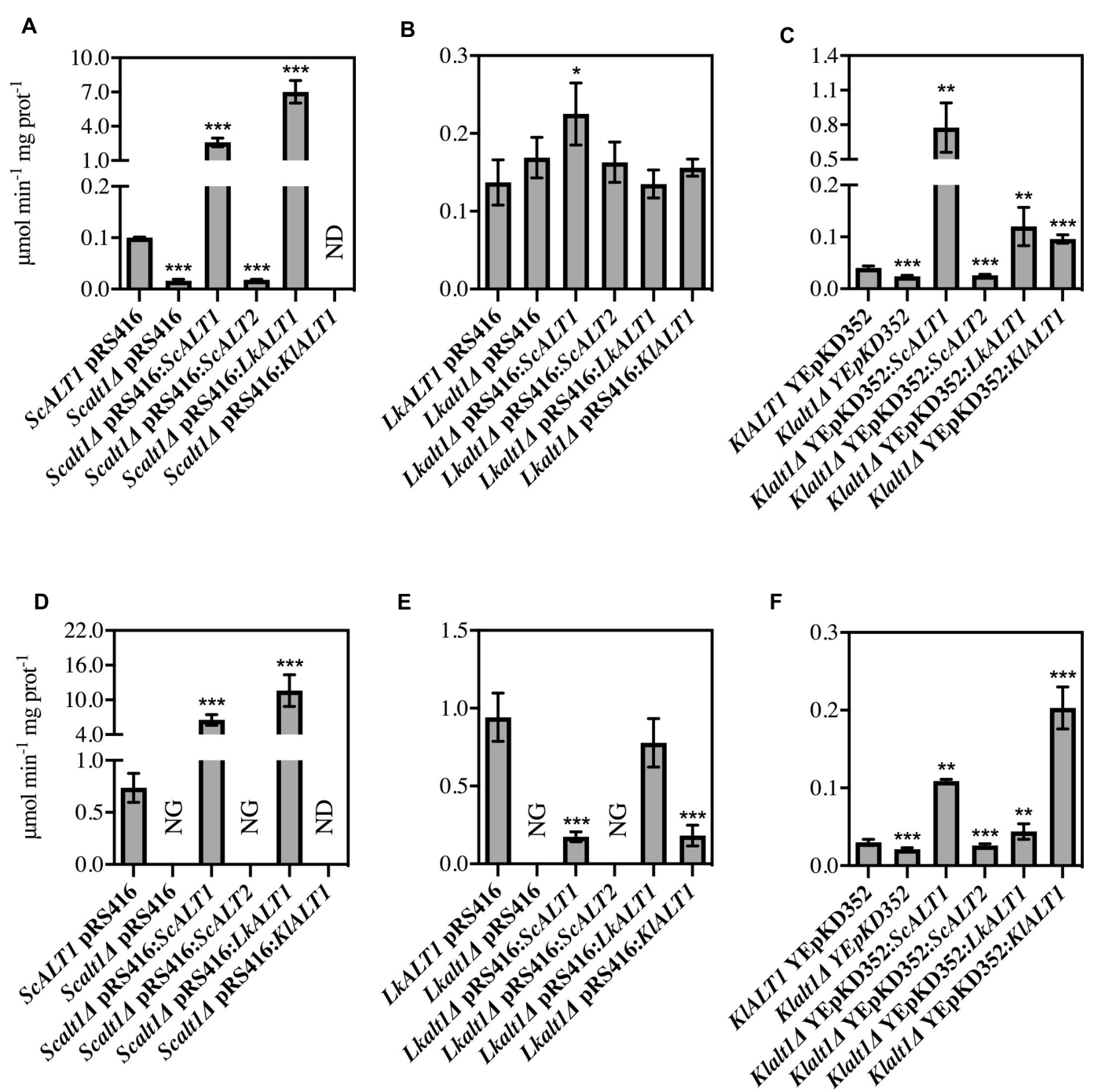

FIGURE 5 | Alanine transaminase specific activity in extracts prepared from complemented strains. Cellular extracts from (A) S. cerevisiae, (B) L. Kluyveri, and (C) K. lactis in glucose- ammonium sulfate or (D-F) in glucose-alanine grown cultures. Specific activity is expressed as $\mu \mathrm{mol} \mathrm{min}^{-1} \mathrm{mg}$ of protein ${ }^{-1}$, values are presented as means \pm SD from three independent experiments. NG, no growth; ND, not detected. The asterisks indicate significantly different ${ }^{*} p<0.1$; $\left.{ }^{* *} p<0.05 ; * p<0.01\right)$ compared with the wt control.

sole nitrogen source, extracts from Lkalt1 $\Delta$ transformed with an empty plasmid, presented equivalent alanine transaminase specific activity to that found in extracts of its corresponding wild type strain (Figure 5B). As observed before, in L. kluyveri alanine transaminase activity was not exclusively displayed by LkAlt1 (Table 2). Thus, the activity measured under this condition, in all the Lkalt $1 \Delta$ transformed strains, is the result of the combined activities of the heterologous enzyme and the alternative alanine transaminase. Even though this other transaminase activity is also present in extracts of Lkalt1 $\Delta$ grown in alanine, its contribution is minor, not allowing growth of Lkalt1 $\Delta$ carrying pRS416 empty plasmid. In this medium extracts from $L k a l t 1 \Delta$ transformed with LkALT1 presented an activity similar to that detected in extracts obtained from the wild type strain, while those obtained from Lkalt1 $\Delta$ transformed with ScALT1 or KlALT1, presented a four times lower activity than the one observed in the wild type strain (Figure 5E), consistent with the partial complementation phenotype of these strains (Figure 3B). The activity in extracts obtained from glucose-ammonium sulfate or glucose-alanine of Klalt1 $\Delta$ transformed with ScALT1, LkALT1, or KlALT1 was higher than in the wild type. All the mutants transformed with plasmids harboring ScALT2 did not display activity as well as the negative controls (Figures 5A-F), indicating ScALT2 incapacity to complement ScALT1, LkALT1, and KlALT1 (Figures 3, 4). This result was expected since we had previously observed that even when ScALT2 is over-expressed using a TET promoter, higher production of $S c A l t 2$ protein does not lead to detectable activity (Peñalosa-Ruiz et al., 2012). Increased activity of some samples, due to plasmid copy number, as compared to their respective wild type strain was not followed by an increased alanine pool 


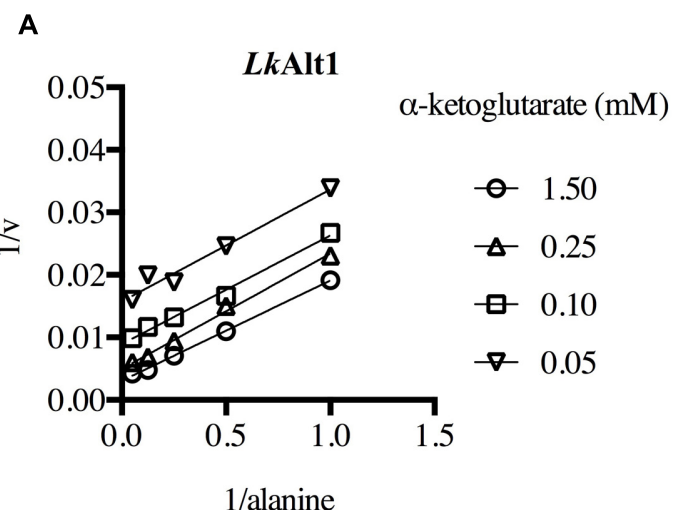

B

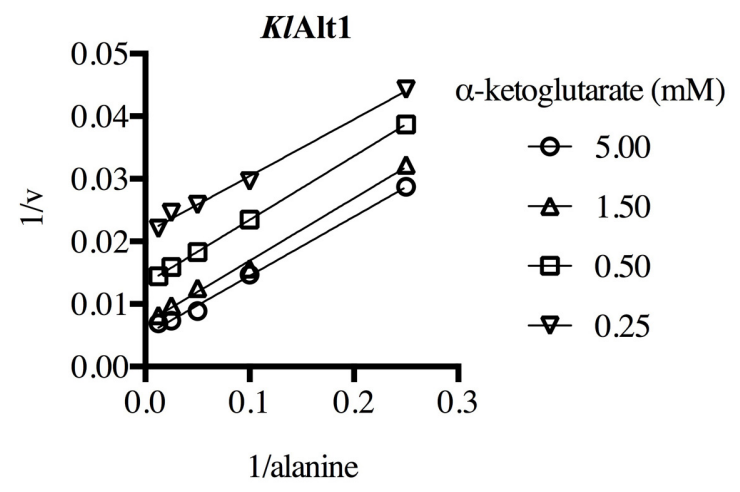

C

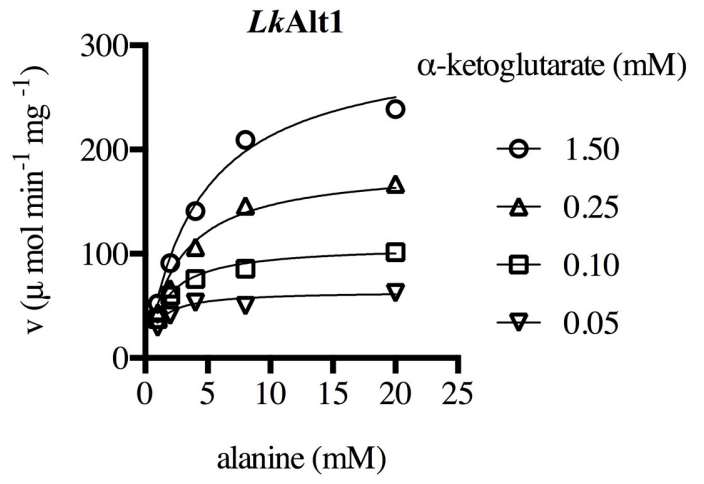

D

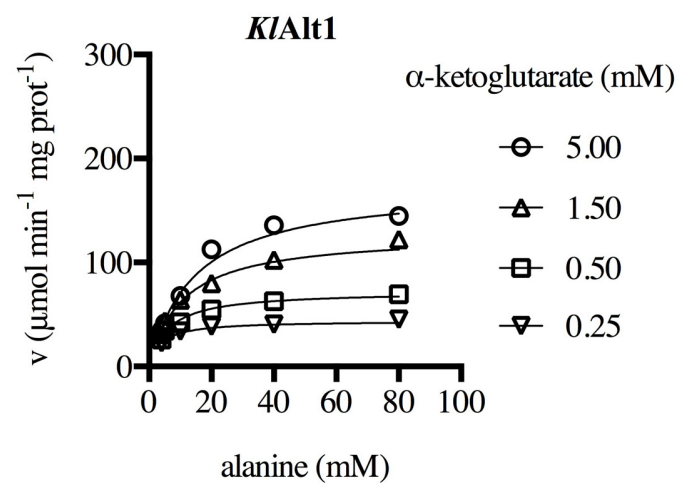

FIGURE 6 | LKAlt1 and KIAlt1 are alanine transaminases with the characteristic ping pong mechanism. Initial velocities were measured at different alanine and $\alpha$-ketoglutarate concentrations. Double reciprocal plots of (A) LkAlt1 and (B) KIAlt1. Global fit of (C) LkAlt1 and (D) KIAlt1 to the ping pong equation. The corresponding kinetic parameters are shown in Table 3.

or higher growth rate (Figures 3, 4), indicating that an increase in the enzyme concentration, does not result in higher alanine production, suggesting that in vivo these enzymes, work far from their Vmax, so increased enzyme concentration does not result in a higher flux through this enzymes.

\section{LkAlt1 and KIAlt1 Display Similar Kinetic Parameters and a Ping Pong Mechanism}

To investigate LkAlt1 and KlAlt1 kinetic properties, His-tagged enzymes were purified to electrophoretic homogeneity after heterologous over-expression in E. coli. Denaturing SDS-PAGE showed a molecular mass of about $50 \mathrm{kDa}$ for the two enzymes, in agreement with the value deduced from their amino acid sequences, which were 61.16 and $59.23 \mathrm{kDa}$ for LkAlt1 and KlAlt1, respectively (Supplementary Figure S1). The oligomeric structures revealed by light scattering of the purified samples (LkAlt1 $100.1 \pm 10.8 \mathrm{kDa}$ and KlAlt1 $135.9 \pm 25.8 \mathrm{kDa}$ ) indicate that the active form of both enzymes is an homodimer. Enzymatic activity of the pure enzymes was measured in Tris$\mathrm{HCl}$ buffer at $\mathrm{pH}$ values ranging from 5.5 to 8.5 , maximum activity was obtained around $\mathrm{pH} 7$ for the two enzymes. It is worth mentioning that $L k$ Alt1 was sensitive to acidic $\mathrm{pH}$ while KlAlt1 was sensitive to basic $\mathrm{pH}$ (Supplementary Figure S2).
Initial velocity measurements were made at different $\alpha$-ketoglutarate and alanine concentrations. The resulting double reciprocal plot pattern obtained for both enzymes, corresponded to that expected for enzymes with a Ping-Pong mechanism (Figures 6A,B). Thus, the data were globally fitted to a Ping-Pong equation (Figures 6C,D). The resulting kinetic parameters for LkAlt1 and KlAlt1 are presented in Table 3. It follows that the orthologous proteins $L k$ Alt1 and KlAlt1 are alanine transaminases with similar $k_{\text {cat }}$, and $k_{\mathrm{m}}$, in both cases the affinity for $\alpha$-ketoglutarate was higher than the one for alanine (Table 3). Results from our laboratory indicate that $S$. cerevisiae ScAlt1 is also an alanine transaminase (Peñalosa-Ruiz et al., 2012) with close kinetic parameters to the ones observed in L. kluyveri LkAlt1 and K. lactis KlAlt1 (unpublished results).

TABLE 3 | Kinetic parameters of L. Kluyveri LkAlt1 and K. lactis KIAlt1.

\begin{tabular}{lcccc}
\hline Enzyme & $\boldsymbol{k}_{\text {cat }}\left(\mathbf{s}^{-\mathbf{1}}\right)$ & $\boldsymbol{k}_{\mathrm{a}}(\mathbf{m M})$ & $\boldsymbol{k}_{\mathrm{b}}(\mathbf{m M})$ & $\boldsymbol{R}^{\mathbf{2}}$ \\
\hline LkAlt1 & 355 & 4.88 & 0.22 & 0.98 \\
KIAlt1 & 203 & 17.25 & 0.92 & 0.98
\end{tabular}

Data were fitted to the ping pong equation. $k_{a}$ represents the $k_{m}$ for alanine and $k_{b}$ the $k_{m}$ for $\alpha$-ketoglutarate. 


\section{Divergent Expression Profile between LKALT1 and KIALT1 Is Independent of Chromatin Organization}

In S. cerevisiae, ScALT1 and ScALT2 are differentially expressed. Previous analysis revealed that the expression profile of ScALT1 resembles that of a catabolic gene, whose expression is induced in the presence of alanine. Opposed expression pattern is observed for ScALT2, which is repressed whenever alanine is present (Peñalosa-Ruiz et al., 2012). To investigate whether the expression pattern of LkALT1 and KlALT1 was similar to that of $S c A L T 1$ or $S c A L T 2$, Northern blot analysis were carried out in samples obtained from wild type strain cultures in different growth phases on either glucose-ammonium sulfate or glucose-alanine. $L k A L T 1$ was repressed in a medium with ammonium sulfate as sole nitrogen source and induced when alanine was the nitrogen source (Figure 7A), mirroring ScALT1 expression profile (Peñalosa-Ruiz et al., 2012). KlALT1 showed constitutive expression, was equally expressed in both glucose-ammonium sulfate and glucose-alanine conditions (Figure 7C). Enzymatic activity followed the expression profile observed for the two orthologous genes LkALT1 and KlALT1 (Table 2).

To analyze LkALT1 and KlALT1 promoter organization, nucleosome scanning assay (NuSA) was preformed. Samples obtained from exponential phase growth cultures $\left(\mathrm{OD}_{600} \mathrm{~nm}\right.$ 0.3) on ammonium sulfate or alanine as sole nitrogen sources were analyzed to determine nucleosome positioning across the promoters of both genes. After yeast DNAnucleosome interaction was fixated with formaldehyde, cells were spheroplasted and incubated with MNase. The resulting DNA fragments obtained after MNase digestion were analyzed by quantitative PCR (qPCR). For normalization L. kluyveri LkVCX1 locus (SAKL0F09878g), or K. lactis KlVCX1 locus (KLLA-ORF439) (Infante et al., 2011) were used. Nucleosome positioning was equivalent for LkALT1 and KlALT1 promoters when cells were grown on ammonium sulfate or alanine (Figures 7B,D), indicating that the different expression profiles observed for the LkALT1 promoter on ammonium sulfate or alanine was not related to nucleosome organization (Figure 7A). As expected for KlALT1, which displays constitutive expression, NuSA analysis showed similar nucleosome organization on both growth conditions (Figure 7D). Since the nucleosome positioning did not change in either one of the promoters tested it can be hypothesized that the most likely transcriptional factors involved in LkALT1 and KlALT1 transcriptional regulation should have their binding sites in the Nucleosome Free Region (NFR). Accordingly, an "in silico" analysis of the LkALT1 and KlALT1 promoter sequences was performed ${ }^{1}$, considering the consensus and conserved binding elements known for $S$. cerevisiae. In both promoters, cis elements for Gcn4 and Rtg3 in either free or nucleosome protected regions were found, additionally, in KlALT1 promoter region an Nrg1 sequence was found. This analysis suggests that LkALT1 could be regulated by the combined action of Gcn 4

${ }^{1}$ www.yeastract.com and Rtg3 orthologous transcriptional factors, while that of KlALT1 would also combine Nrg1. Further research on this matter will be required to determine whether the predicted regulators are involved in $L k A L T 1$ and KlALT1 transcriptional regulation.

\section{DISCUSSION}

Here, we present a combined phenotypic, biochemical and transcriptional regulation analysis of the L. kluyveri LkALT1 and $K$. lactis KlALT1 genes, which shows that both encode transaminases involved in alanine biosynthesis and catabolism. Interestingly, the $S$. cerevisiae isozyme encoded by ScALT1, retained alanine transaminase activity, while ScALT2 encoded enzyme did not conserve this activity (Peñalosa-Ruiz et al., 2012). In all three cases transaminase activity followed gene expression levels of their encoding genes. Accordingly, while ScALT1, LkALT1 and KlALT1, were capable of reciprocally complementing their role in alanine metabolism, ScALT2 was not. Moreover, it was discovered that LkALT1 and KlALT1 harbors an additional function besides that of alanine transaminase, which was neither retained by ScALT1 nor ScALT2.

\section{Insights into the Evolution of Alanine Metabolism}

Of the three yeasts, which were used as experimental models in this work, $K$. lactis was the first one to diverge from the linage leading to S. cerevisiae (Merico et al., 2007; Huerta-Cepas et al., 2014; Marcet-Houben and Gabaldón, 2015), thus it can be considered that this yeast is an appropriate representative of KLE progenitor physiology. In K. lactis alanine metabolism is accomplished by more than one pathway; trough the KlAlt1 alanine transaminase, and must likely by another yet unidentified alanine transaminase. Nevertheless, the existence of alternative pathways involved in alanine metabolism cannot be ruled out for example glutamine transaminase, which is known to exist in S. cerevisiae (Soberón and González, 1987), that could support alanine biosynthesis from pyruvate and glutamine. In $K$. lactis, the expression of KlALT1 and the activity displayed by the encoded protein is equivalent under biosynthetic or catabolic conditions. Also, the KlAlt1 alanine transaminase activity detected in a Klalt1 $\Delta$ mutant shows similar enzymatic activity in both conditions, suggesting that KlAlt1 is similarly specialized in alanine biosynthesis and catabolism. Nevertheless, while a Klalt1 $\Delta$ strain displays wild type growth rate under biosynthetic conditions, it shows decreased growth rate when grown on alanine as sole nitrogen source, indicating that the alternative pathways involved in alanine metabolism cannot fully compensate KlAlt1catabolic role.

As well as K. lactis, L. kluyveri can also be considered a $K L E$ ancestral type yeast. In $L$. kluyveri, alanine biosynthesis and catabolism can be achieved by more than one pathway, since an Lkalt1 $\Delta$ mutant is able to grow on ammonium or alanine as sole nitrogen sources. In L. kluyveri LkALT1 expression is increased when alanine is provided as sole nitrogen source, 

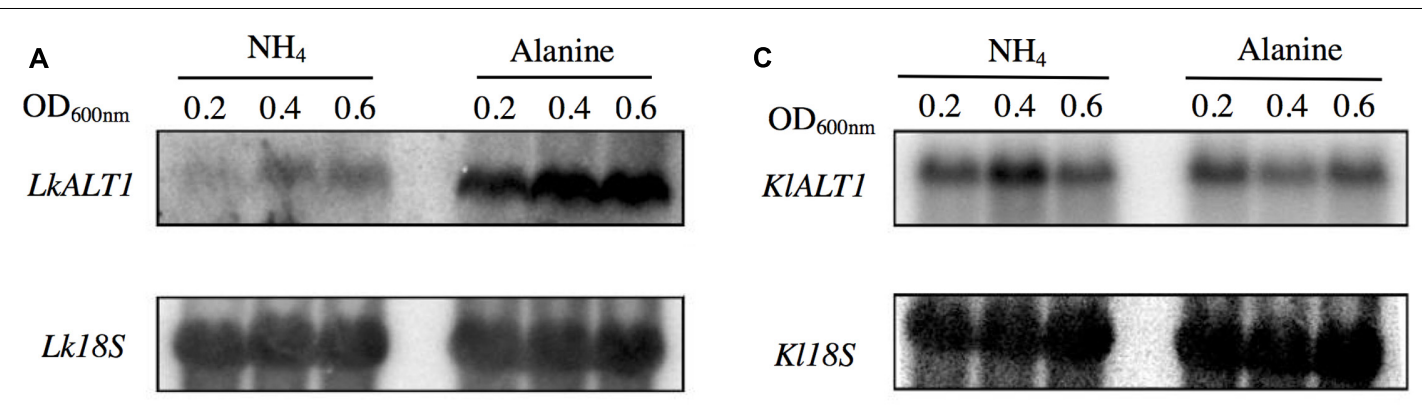

Kl18S

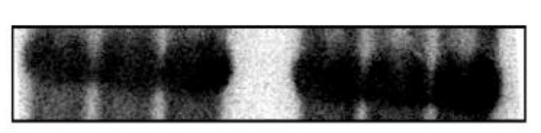

B
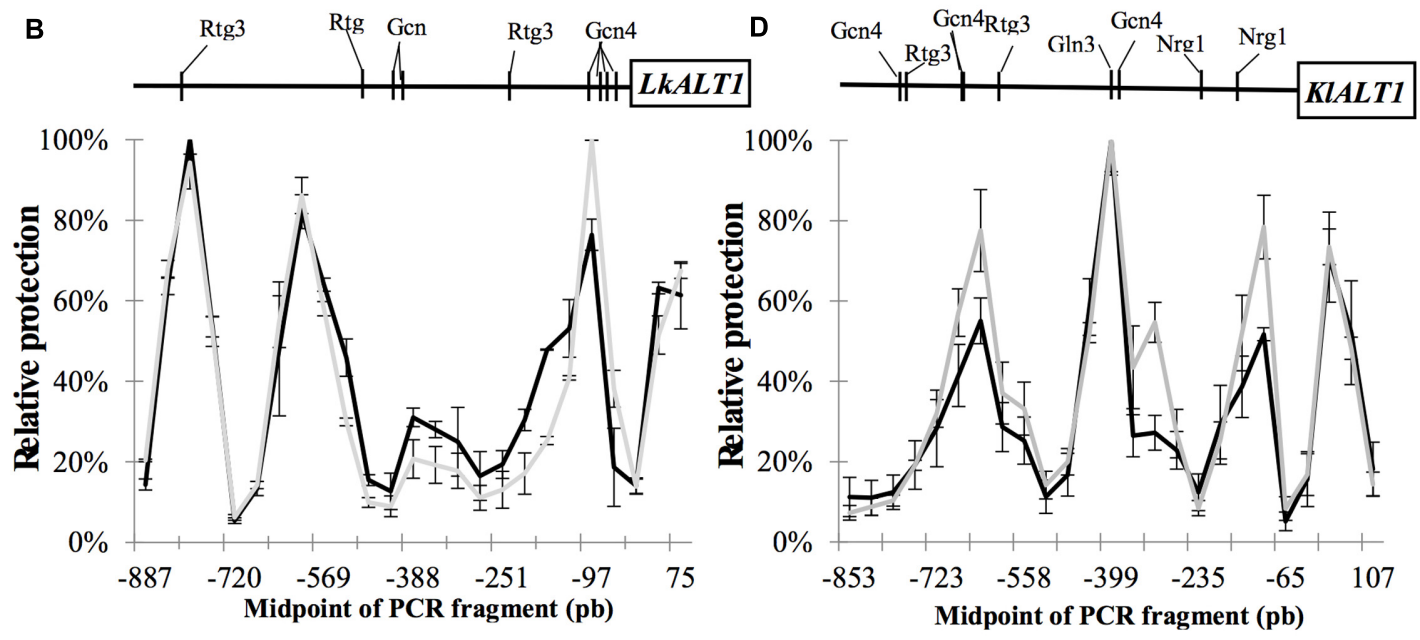

Ammonium

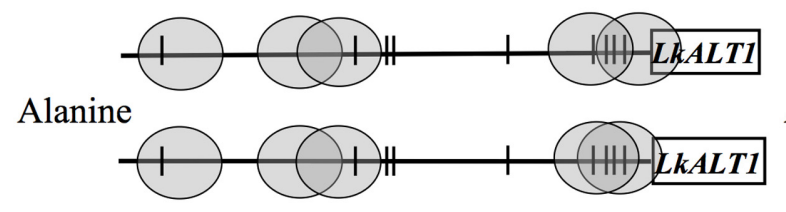

Ammonium

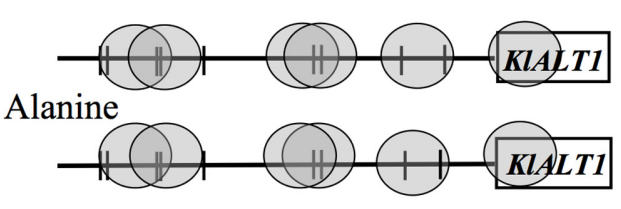

FIGURE 7 | LKALT1 and KIALT1 expression profile and NuSA assay. Northern blot analysis shown expression profile of $L K A L T 1$ (A) and KIALT1 (B), in both cases $L K 18 S$ or KI18S ribosomal RNA was used as control in glucose-amoniumm sulfate or glucose alanine. (C) Nucleosome scanning assay (NuSA) $L K A L T 1$ gene promoter in glucose-ammonium (black line) or glucose-alanine (gray line); and (D) Nucleosome Scanning Assay (NuSA) KIALT1 gene promoter in glucose-ammonium (black line) or glucose-alanine (gray line); nucleosomes are shown in gray ovals and vertical lines shown DNA binding sites. Error bars represent the standard deviations from two independent experiments.

which results in higher alanine transaminase activity, constituting a specialization of $L k$ Alt1 catabolic role. In addition, under this condition, the LkAlt1 independent alanine transaminase decreases its activity, enhancing the leading role of LkAlt1 in alanine catabolism. The expression pattern observed in LkALT1, favoring its catabolic role, was maintained by ScALTI, but the degree of specialization in alanine catabolism increased to the extent in which ScAlt1 is the only enzyme capable of catabolizing alanine, since the alternative pathway for alanine catabolism present in K. lactis and L. kluyveri was lost in S. cerevisiae. It is not surprising that the specialization of ScAlt1 and LkAlt1 towards alanine catabolism was achieved through a change in gene expression pattern, since the reaction catalyzed by transaminases, is close to equilibrium, and thus the direction of the reaction largely depends on substrates and products concentration, and given the enzymatic mechanism of the reaction, modification in the kinetic parameters of the enzyme would affect in the same proportion the biosynthetic and catabolic direction of the reaction (Nisonoff et al., 1953). It could be considered that the catabolic specialization present in these enzymes is compelling in order to improve their anaplerotic character which enables them to furnish pyruvate to the tricarboxilic acid cycle (Land, 2002). It can be considered that the anaplerotic role of alanine transaminase is less compelling for $K$. lactis than for S. cerevisiae and L. kluyveri, since K. lactis metabolism is constitutively respiratory (Breunig et al., 2000) and S. cerevisiae and L. kluyveri have a facultative metabolism (Moller et al., 2002; Merico et al., 2007). Hence, L. kluyveri and S. cerevisiae catabolic specialization arrived to the extreme in which $S$. cerevisiae lost the alternative pathways for alanine catabolism present in 
both L. kluyveri and K. lactis. However, increased catabolic character did not affect the prominent biosynthetic role played by these three enzymes, which contribute with at least half of the alanine pool, even when more than one pathway for alanine biosynthesis is present in the three species. This redundancy is also found in E. coli, where alanine is synthesized by three different transaminases, and only the triple mutant is an alanine auxotroph (Yoneyama et al., 2011). In this regard, it is important to consider that in E. coli alanine is the second amino acid with highest intracellular concentration, and in Neurospora and S. cerevisiae it is the amino acid displaying highest intracellular concentration (Yuan et al., 2006; Canelas et al., 2008; Kim et al., 2011). Although no information on free intracellular amino acid pools exists for L. kluyveri and K. lactis, results presented in this paper indicate that alanine could constitute one of the highest accumulated amino acids in these yeasts. These observations support the proposition that alanine could play an important role in addition to that as protein building block as has been shown for other amino acids (Stephen and Jamieson, 1997; Takagi et al., 1997; Crespo and Hall, 2002; Eisler et al., 2004; Binda et al., 2009).

\section{ScALT2 Evolutionary History}

Although the closest taxonomic group of ScALT2 parental strain is the KLE clade, ScAlt2 is not an alanine transaminase, nor displays the alternative function encoded by LkALT1 and KlALT1. A structural analysis comparing $S c$ Alt1 and $S c$ Alt2 sequences demonstrated that ScAlt2 has conserved the binding site for alanine and for pyridoxal-5-phospate which is the coenzyme which enables transaminase activity (García-Campusano, 2009). Together, these data indicate that in the KLE parental strain the ancestral form of ScALT2 codified for an alanine transaminase. Thus, it can be considered that when the hybrid was formed the KLE parental strain contributed with at least two alanine transaminases, one of them endosed by the ancestral form of ScALT2, and another one codified by a yet unidentified gene encoding the observed transaminase activity present in the Lkalt $1 \Delta$ and Klalt $1 \Delta$ mutants. In addition, the $Z T$ parental strain provided a third alanine transaminase, which corresponded to the ScALT1 ancestral form, which probably was already specialized towards alanine catabolism through gene expression, since this tendency is already present in L. kluyveri LkALT1. The specialization of ScALT1 towards alanine catabolism and its contribution to alanine biosynthesis, not only relaxed the selective pressure that would favor the maintenance of an ScALT1 and ScALT2 independent alternative catabolic pathways, provided by the KLE parental, but also flexibilized the need to conserve ScALT2 encoded alanine transaminase activity. Hence, the divergence between $S c A L T 1$ and $S c A L T 2$ can be explained by loss-of-function, sub-functionalization, and neofunctionalization models posed to explain the fate of duplicated genes. The scenario provided by the loss-of-function model, is the most unlikely, since ScALT2 promoter has retained the capacity to respond to alanine concentration and a complete soluble protein with no transaminase activity is synthesized (Peñalosa-Ruiz et al., 2012). If the divergence of ScALT1 and ScALT2 had occurred through subfunctionalization, one would expect that the transaminase activity present in $L k A l t 1$ and in KlAlt1 would have only been retained by $S c A l t 1$ and lost by $S c A l t 2$, as shown in this work; whereas, the alternative function provided by $L k A L T 1$ and KlALT1 would have been delegated to ScALT2 and lost from ScALT1, which is not consistent with the finding that ScAlt2 does not complement neither Lkalt1 $\Delta$ nor Klalt1 $\Delta$ mutants. Finally, under a neofunctionalization model, it would be expected that ScAlt2 function would be absent from either ScALT1 LkALT1 or KlALT1. The data herein presented support this later scenario; nevertheless, further studies are needed to understand the role of ScAlt2 and to distinguish between the above presented scenarios.

\section{AUTHOR CONTRIBUTIONS}

AG designed experiments, wrote the MS, obtained funding. XE-F performed and designed experiments, wrote the MS. CC-B performed experiments. MC performed and designed experiments, contributed to MS writing. JG performed experiments. DM performed experiments.

\section{FUNDING}

This study was funded by Dirección General de Asuntos del Personal Académico, UNAM, grant IN201015 (http:// dgapa.unam.mx); Consejo Nacional de Ciencia y Tecnología (CONACYT), grant (CB-2014-239492-B) (http://www.conacyt. gob.mx/), and grant NoCRP/MEX10-03 from the International Center for Genetic Engineering and Biotechnology. XE-F was recipient of a Ph.D. Fellowship from CONACYT. This study was performed in partial fulfillment of the requirements for the Ph.D. degree in Biomedical Sciences of XE-F at the Programa de Doctorado en Ciencias Biomédicas de la Universidad Nacional Autónoma de México, which she carried with a CONACYT doctoral fellowship. The funders had no role in study design, data collection and analysis, decision to publish, or preparation of the manuscript.

\section{ACKNOWLEDGMENTS}

XE-F is a doctoral student from Programa de Doctorado en Ciencias Biomeìdicas, Universidad Nacional Autoìnoma de Meixico (UNAM) and received a doctoral fellowship from CONACYT. We thank Juan Pablo Pardo and Luis Servín for illuminating discussions during the course of this work, to Mohamed El-Hafidi (Instituto Nacional de Cardiología Ignacio Chávez, Departamento de Biomedicina Cardiovascular, México D. F., México) for amino acid determination. To Héctor Quezada and Toni Gabaldón for critically reading the manuscript, to Beatriz Aguirre for technical support and Rocio Romualdo Martinez for helpful secretarial assistance, to L. Ongay, G. Códiz, and M. Mora (Unidad de Biología Molecular, Instituto de Fisiología Celular, Universidad Nacional Autónoma de México, UNAM) for DNA sequencing and oligonucleotide synthesis and to Mohamed El-Hafidi (Instituto Nacional de Cardiología Ignacio 
Chávez, Departamento de Biomedicina Cardiovascular, México D. F., México) for amino acid determination. This paper is dedicated to Armando Gomez Puyou as a tribute to his lifetime generous dedication to science and teaching.

\section{REFERENCES}

Binda, M., Peli-Gulli, M. P., Bonfils, G., Panchaud, N., Urban, J., Sturgill, T. W., et al. (2009). The Vam6 GEF controls TORC1 by activating the EGO complex. Mol. Cell. 35, 563-573. doi: 10.1016/j.molcel.2009.06.033

Breunig, K. D., Bolotin-Fukuhara, M., Bianchi, M. M., Bourgarel, D., Falcone, C., Ferrero, I. I., et al. (2000). Regulation of primary carbon metabolism in Kluyveromyces lactis. Enzyme Microb. Technol. 26, 771-780. doi: 10.1016/ S0141-0229(00)00170-8

Canelas, A. B., Ras, C., ten Pierick, A., van Dam, J. C., Heijnen, J. J., and van Gulik, W. M. (2008). Leakage-free rapid quenching technique for yeast metabolomics. Metabolomics 4, 226-239. doi: 10.1007/s11306-008-0116-4

Colón, M., Hernandez, F., Lopez, K., Quezada, H., Gonzalez, J., Lopez, et al. (2011). Saccharomyces cerevisiae Bat1 and Bat2 aminotransferases have functionally diverged from the ancestral-like Kluyveromyces lactis orthologous enzyme. PLOS ONE 6:e16099. doi: 10.1371/journal.pone.0016099

Conrad, B., and Antonarakis, S. E. (2007). Gene duplication: a drive for phenotypic diversity and cause of human disease. Аnnu. Rev. Genomics Hum. Genet. 8, 17-35. doi: 10.1146/annurev.genom.8.021307.110233

Crespo, J. L., and Hall, M. N. (2002). Elucidating TOR signaling and rapamycin action: lessons from Saccharomyces cerevisiae. Microbiol. Mol. Biol. Rev. 66, 579-591. doi: 10.1128/MMBR.66.4.579-591.2002

Eisler, H., Frohlich, K. U., and Heidenreich, E. (2004). Starvation for an essential amino acid induces apoptosis and oxidative stress in yeast. Exp. Cell Res. 300, 345-353. doi: 10.1016/j.yexcr.2004.07.025

Espinosa-Cantú, A., Ascencio, D., Barona-Gómez, F., and DeLuna, A. (2015). Gene duplication and the evolution of moonlighting proteins. Front. Genet. 6:227. doi: 10.3389/fgene.2015.00227

García-Campusano, F. T. A. (2009). Estudio functional de los parálogos ALT1 y ALT2 de Saccharomyces cerevisiae Durante el Metabolismo Respiratorio $y$ Fermentativo. Ph.D. thesis, Universidad Nacional Autónoma de México, Mexico City.

Graßl, M., and Supp, M. (1985). “Amino Acids," in Methods of Enzymatic Analysis, ed. H.-U. Bergmeyer (New York, NY: Federal Republic of Germany (VCH)), 345-349.

Huerta-Cepas, J., Capella-Gutierrez, S., Pryszcz, L. P., Marcet-Houben, M., and Gabaldon, T. (2014). PhylomeDB v4: zooming into the plurality of evolutionary histories of a genome. Nucleic Acids Res. 42, D897-D902. doi: 10.1093/nar/ gkt1177

Hughes, A. L. (1994). The evolution of functionally novel proteins after gene duplication. Proc. Biol. Sci. 256, 119-124. doi: 10.1098/rspb.1994. 0058

Infante, J. J., Glynn, L., and Elton, Y. (2011). Chromatin remodeling and protocols. Methods Mol. Biol. 833, 63-87. doi: 10.1007/978-1-61779-477-3_5

Innan, H., and Kondrashov, F. (2010). The evolution of gene duplications: classifying and distinguishing between models. Nat. Rev. Genet. 11, 97-108. doi: $10.1038 / \operatorname{nrg} 2689$

Jorgensen, P., Nishikawa, J. L., Breitkreutz, B. J., and Tyers, M. (2002). Systematic identification of pathways that couple cell growth and division in yeast. Science 297, 395-400. doi: 10.1126/science.1070850

Kellis, M., Birren, B. W., and Lander, E. S. (2004). Proof and evolutionary analysis of ancient genome duplication in the yeast Saccharomyces cerevisiae. Nature 428, 617-624. doi: 10.1038/nature02424

Kim, J. D., Kaiser, K., Larive, C. K., and Borkovich, K. A. (2011). Use of ${ }^{1} \mathrm{H}$ nuclear magnetic resonance to measure intracellular metabolite levels during growth and asexual sporulation in Neurospora crassa. Eukaryot. Cell 10, 820-831. doi: 10.1128/ec.00231-10

Klis, F. M., de Koster, C. G., and Brul, S. (2014). Cell wall-related bionumbers and bioestimates of Saccharomyces cerevisiae and Candida albicans. Eukaryot. Cell 13, 2-9. doi: 10.1128/ec.00250-13

\section{SUPPLEMENTARY MATERIAL}

The Supplementary Material for this article can be found online at: http://journal.frontiersin.org/article/10.3389/fmicb. 2017.01150/full\#supplementary-material

Kondrashov, A. S., Sunyaev, S., and Kondrashov, F. A. (2002). DobzhanskyMuller incompatibilities in protein evolution. Proc. Natl. Acad. Sci. U.S.A. 99, 14878-14883. doi: 10.1073/pnas.232565499

Kondrashov, F. A., and Kondrashov, A. S. (2006). Role of selection in fixation of gene duplications. J. Theor. Biol. 239, 141-151. doi: 10.1016/j.jtbi.2005.08.033

Land, J. M. (2002). "Aspects of skeletal muscle biochemistry in health and disease," in Disorders of Voluntary Muscle, eds G. Karpati, D. Hilton-Jones, and R. C. Griggs (Cambridge: Cambridge University Press), 122-141.

Lowry, O. H., Rosebrough, N. J., Farr, A. L., and Randall, R. J. (1951). Protein measurement with the Folin phenol reagent. J. Biol. Chem. 193, 265-275.

Marcet-Houben, M., and Gabaldón, T. (2015). Beyond the whole-genome duplication: phylogenetic evidence for an ancient interspecies hybridization in the baker's yeast lineage. PLoS Biol. 13:e1002220. doi: 10.1371/journal.pbio. 1002220

Merico, A., Sulo, P., Piskur, J., and Compagno, C. (2007). Fermentative lifestyle in yeasts belonging to the Saccharomyces complex. FEBS J. 274, 976-989. doi: 10.1111/j.1742-4658.2007.05645.x

Moller, K., Christensen, B., Forster, J., Piskur, J., Nielsen, J., and Olsson, L. (2002). Aerobic glucose metabolism of Saccharomyces kluyveri: growth, metabolite production, and quantification of metabolic fluxes. Biotechnol. Bioeng. 77, 186-193. doi: 10.1002/bit.10122

Montalvo-Arredondo, J., Jiménez-Benítez, A., Colón-González, M., GonzálezFlores, J., Flores-Villegas, M., González, A., et al. (2015). Functional roles of a predicted branched chain aminotransferase encoded by the LkBAT1 gene of the yeast Lachancea kluyveri. Fungal Genet. Biol. 85, 71-82. doi: 10.1016/j.fgb.2015. 11.004

Nisonoff, A., Barnes, F. W. Jr., and Enns, T. (1953). Mechanisms in enzymatic transamination. J. Biol. Chem. 204, 957-970.

Ohno, S. (1970). Evolution by Gene Duplication. Berlin: Springer-Verlag. doi: 10.1007/978-3-642-86659-3

Peñalosa-Ruiz, G., Aranda, C., Ongay-Larios, L., Colon, M., Quezada, H., and Gonzalez, A. (2012). Paralogous ALT1 and ALT2 retention and diversification have generated catalytically active and inactive aminotransferases in Saccharomyces cerevisiae. PLoS ONE 7:e45702. doi: 10.1371/journal.pone.0045702

Quezada, H., Aranda, C., DeLuna, A., Hernandez, H., Calcagno, M. L., MarinHernandez, A., et al. (2008). Specialization of the paralogue LYS21 determines lysine biosynthesis under respiratory metabolism in Saccharomyces cerevisiae. Microbiology 154(Pt 6), 1656-1667. doi: 10.1099/mic.0.2008/017103-0

Segel, I. H. (1975). "Steady-state kinetics of multi-reactant enzymes," in Enzyme Kinetics, ed. I. H. Segel (Hoboken, NJ: John Wiley \& Sons), 606-616.

Sikorski, R. S., and Hieter, P. (1989). A system of shuttle vectors and yeast host strains designed for efficient manipulation of DNA in Saccharomyces cerevisiae. Genetics 122, 19-27.

Sikosek, T., Chan, H. S., and Bornberg-Bauer, E. (2012). Escape from adaptive conflict follows from weak functional trade-offs and mutational robustness. Proc. Natl. Acad. Sci. U.S.A. 109, 14888-14893. doi: 10.1073/pnas.1115620109

Soberón, M., and González, A. (1987). Glutamine degradation through the $\omega$-amidase pathway in Saccharomyces cerevisiae. Microbiology 133, 9-14. doi: 10.1099/00221287-133-1-9

Stephen, D. W., and Jamieson, D. J. (1997). Amino acid-dependent regulation of the Saccharomyces cerevisiae GSH1 gene by hydrogen peroxide. Mol. Microbiol. 23, 203-210. doi: 10.1046/j.1365-2958.1997.2081572.x

Struhl, K., and Davis, R. W. (1981). Transcription of the his3 gene region in Saccharomyces cerevisiae. J. Mol. Biol. 152, 535-552. doi: 10.1016/00222836(81)90267-9

Takagi, H., Iwamoto, F., and Nakamori, S. (1997). Isolation of freeze-tolerant laboratory strains of Saccharomyces cerevisiae from proline-analogue-resistant mutants. Appl. Microbiol. Biotechnol. 47, 405-411. doi: 10.1007/s00253 0050948 
Tang, Y. C., and Amon, A. (2013). Gene copy-number alterations: a cost-benefit analysis. Cell 152, 394-405. doi: 10.1016/j.cell.2012.11.043

Wolfe, K. H., and Shields, D. C. (1997). Molecular evidence for an ancient duplication of the entire yeast genome. Nature 387, 708-713. doi: 10.1038/ 42711

Yoneyama, H., Hori, H., Lim, S. J., Murata, T., Ando, T., Isogai, E., et al. (2011). Isolation of a mutant auxotrophic for L-alanine and identification of three major aminotransferases that synthesize L-alanine in Escherichia coli. Biosci. Biotechnol. Biochem. 75, 930-938. doi: 10.1271/bbb.100905

Yuan, J., Fowler, W. U., Kimball, E., Lu, W., and Rabinowitz, J. D. (2006). Kinetic flux profiling of nitrogen assimilation in Escherichia coli. Nat. Chem. Biol. 2, 529-530. doi: 10.1038/nchembio816
Conflict of Interest Statement: The authors declare that the research was conducted in the absence of any commercial or financial relationships that could be construed as a potential conflict of interest.

Copyright (C) 2017 Escalera-Fanjul, Campero-Basaldua, Colón, González, Márquez and González. This is an open-access article distributed under the terms of the Creative Commons Attribution License (CC BY). The use, distribution or reproduction in other forums is permitted, provided the original author(s) or licensor are credited and that the original publication in this journal is cited, in accordance with accepted academic practice. No use, distribution or reproduction is permitted which does not comply with these terms. 